OPEN ACCESS

Edited by:

Ana Traven,

Monash University, Australia

Reviewed by:

Elizabeth R. Ballou,

University of Birmingham,

United Kingdom

Megan Lenardon,

University of New South Wales,

Australia

*Correspondence:

Pascale Cotton

pascale.cotton@univ-lyon1.fr

Specialty section

This article was submitted to

Fungi and Their Interactions,

a section of the journal

Frontiers in Microbiology

Received: 29 October 2018 Accepted: 08 February 2019

Published: 25 February 2019

Citation:

Laurian R, Dementhon $K$, Doumèche $B$, Soulard A, Noel $T$,

Lemaire $M$ and Cotton P (2019)

Hexokinase and Glucokinases Are Essential for Fitness and Virulence in the Pathogenic Yeast Candida albicans. Front. Microbiol. 10:327. doi: 10.3389/fmicb.2019.00327

\section{Hexokinase and Glucokinases Are Essential for Fitness and Virulence in the Pathogenic Yeast Candida albicans}

\author{
Romain Laurian', Karine Dementhon', Bastien Doumèche ${ }^{3}$, Alexandre Soulard', \\ Thierry Noel ${ }^{2}$, Marc Lemaire ${ }^{1}$ and Pascale Cotton ${ }^{1 *}$ \\ ${ }^{1}$ Génétique Moléculaire des Levures, UMR-CNRS 5240 Microbiologie Adaptation et Pathogénie, Université de Lyon - \\ Université Lyon 1, Lyon, France, ${ }^{2}$ Laboratoire de Microbiologie Fondamentale et Pathogénicité, UMR-CNRS 5234, \\ Université de Bordeaux, Bordeaux, France, ${ }^{3}$ Institut de Chimie et Biochimie Moléculaires et Supramoléculaires, Université \\ de Lyon - Université Lyon 1, Lyon, France
}

The pathogenic yeast Candida albicans is both a powerful commensal and a pathogen of humans that can infect wide range of organs and body sites. Metabolic flexibility promotes infection and commensal colonization by this opportunistic pathogen. Yeast cell survival depends upon assimilation of fermentable and non-fermentable locally available carbon sources. Physiologically relevant sugars like glucose and fructose are present at low levels in host niches. However, because glucose is the preferred substrate for energy and biosynthesis of structural components, its efficient detection and metabolism are fundamental for the metabolic adaptation of the pathogen. We explored and characterized the C. albicans hexose kinase system composed of one hexokinase (CaHxk2) and two glucokinases (CaGlk1 and CaGlk4). Using a set of mutant strains, we found that hexose phosphorylation is mostly performed by CaHxk2, which sustains growth on hexoses. Our data on hexokinase and glucokinase expression point out an absence of cross regulation mechanisms at the transcription level and different regulatory pathways. In the presence of glucose, CaHxk2 migrates in the nucleus and contributes to the glucose repression signaling pathway. In addition, CaHxk2 participates in oxidative, osmotic and cell wall stress responses, while glucokinases are overexpressed under hypoxia. Hexose phosphorylation is a key step necessary for filamentation that is affected in the hexokinase mutant. Virulence of this mutant is clearly impacted in the Galleria mellonella and macrophage models. Filamentation, glucose phosphorylation and stress response defects of the hexokinase mutant prevent host killing by C. albicans. By contributing to metabolic flexibility, stress response and morphogenesis, hexose kinase enzymes play an essential role in the virulence of $C$. albicans.

Keywords: Candida albicans, hexokinase, glucokinase, glycolysis, glucose repression, hyphal transition, virulence 


\section{INTRODUCTION}

C. albicans is an opportunistic pathogen which exists in a relatively harmless state in the microbiota of healthy individuals. It is notably present on the mucosal surfaces composing the digestive tract (Odds, 1988; Calderone and Clancy, 2012). Perturbations of the normal microbiota, use of medical implants, or predisposing factors like diabetes can trigger $C$. albicans infection. C. albicans is the most common cause of fungal nosocomial infections associated with high mortality rates in immunocompromised patients (Jarvis and Martone, 1992; Perlroth et al., 2007; Armstrong-James et al., 2014).

C. albicans colonizes diverse host microenvironments such as skin, mucosa, blood, and organs, (Odds, 1988). Among the wide range of virulence traits, survival at $37^{\circ} \mathrm{C}, \mathrm{pH}$ and osmolarity adaptation, secretion of lytic enzymes, alteration of the immune response, morphological changes, such as a transition between yeast and hyphae, occur during infection and promote host invasion (Noble et al., 2017). Another crucial factor is the metabolic capacity to assimilate host nutrients. The importance of metabolic flexibility to promote systemic infection and commensal colonization has been clearly emphasized during the past years (Wilson et al., 2009; Sandai et al., 2012; Brown et al., 2014; Childers et al., 2016). Genomic tools revealed that rapid transcriptional responses take place to set up a niche-specific carbon metabolism (Lorenz et al., 2004; Barelle et al., 2006; Bonhomme et al., 2011; Ene et al., 2012, 2013; Brown et al., 2014). Utilization of alternative non-fermentable sources through the glyoxylate and gluconeogenesis pathways is essential to support C. albicans proliferation in vivo (Lorenz and Fink, 2001; Miramón and Lorenz, 2017). However, physiologically relevant hexose sugars like glucose, galactose, and fructose are transiently available at low level in the gastrointestinal tract and only glucose $(0.05-0.1 \%)$ is present in the bloodstream (Barelle et al., 2006; Pérez et al., 2013; Miramón and Lorenz, 2017). During survival in blood, invasion of kidneys and liver, expression of C. albicans infection-associated genes involved in glycolysis has been reported (Brown et al., 2007; Wilson et al., 2009; Pérez et al., 2013). Complete glycolytic activation by the two key transcriptional regulators Gal4p and Tye7p is required for full virulence in Galleria mellonella and mice (Askew et al., 2009). Glucose is the preferred substrate for ATP generation, metabolic precursors synthesis and maintenance of a reductive potential in eukaryotes (Flores et al., 2000; Rolland et al., 2001). Hence, accurate and efficient glucose detection and metabolization pathways constitute a fundamental basis for metabolic adaptation of the pathogen. In C. albicans, there are 20 predicted glucose transporters; one of them, CaHgt4, a high affinity sensor of the SRR pathway (Sugar Receptor Repressor), is essential for low glucose level induction of six of the C. albicans transporters. In addition, CaHgt4 is also required for filamentation and contributes to virulence in mice (Brown et al., 2006; Sabina and Brown, 2009).

Once detected, the initial step in glucose utilization is its transport and following transformation into a sugar phosphate. Most fungi contain at least two active hexose kinases: glucokinase and hexokinase. In $S$. cerevisiae, the hexokinase Hxk2 is the predominant glucose kinase in cells growing in high glucose conditions (Herrero et al., 1995). Both enzymes can support growth on glucose but hexokinases and glucokinases can also phosphorylate other hexoses like fructose and mannose (Cárdenas et al., 1998). The enzymatic equipment for hexose phosphorylation varies among different yeasts, although no physiological explanation for the differences has been found. A search in the genome of $C$. albicans revealed the presence of two hexokinases (CaHXK1 and CaHXK2) and two glucokinase genes (CaGLK1 and CaGLK4). The hexokinase $\mathrm{CaHxk1}$ does not phosphorylate glucose but $N$-acetylglucosamine (GlcNAc), an extracellular carbon source present in the mucous membranes, triggering the transition between yeast and hyphal form (Yamada-Okabe et al., 2001; Rao et al., 2013). However, the hexokinase CaHxk2 and the glucokinases $\mathrm{CaGlk} 1$ and $\mathrm{CaGlk} 4$ have not been characterized so far and their respective role in $C$. albicans fitness and virulence has not been investigated yet. Moreover, nothing is known about the enzymatic functions of $\mathrm{CaHxk} 2, \mathrm{CaGlk} 1$ and $\mathrm{CaGlk} 4$ and their putative dual regulatory role in glucose repression. The preferential use of glucose by yeasts results from glucose-induced transcriptional repression via the CaSnf1, essential AMP-kinase, which phosphorylates the transcription factor CaMig1 (Zaragoza et al., 2000; Corvey et al., 2005). Based on the $S$. cerevisiae model, CaMig1 should form a necessary complex with the hexokinase $\mathrm{CaHxk} 2$ to shuttle in the nucleus and generate the glucose repression signal (Moreno et al., 2005), but this particular step has not been described yet in C. albicans.

In this study, we evaluated the contribution of CaHxk2, $\mathrm{CaGlk} 1$ and $\mathrm{CaGlk} 4$ to the phosphorylation of hexoses and to the glucose repression process. Substantial insights in the functional consequences of hexokinase and/or glucokinases deficiency for C. albicans growth, various stress responses, morphological transition and virulence are also proposed.

\section{MATERIALS AND METHODS}

\section{Strains and Growth Conditions}

C. albicans strains used in this study are listed in Supplementary Table S1. Strains were grown at $30^{\circ} \mathrm{C}$ or $37^{\circ} \mathrm{C}$ on YPG medium (1\% yeast extract, $2 \%$ peptone, $2 \%$ glucose). When necessary glucose was added at various concentrations (from 0.01 to $2 \%$ ) or replaced by other carbon sources like $2 \%$ glycerol or $2 \%$ lactate. To analyze the influence of the carbon source on CaHXK2 and CaGLK1/4 transcript level, early log phase cells grown in lactate $2 \%(\mathrm{OD}=1.8)$ were transferred to the different media containing $2 \%$ lactate, $0.1 \%$ or $2 \%$ glucose, $2 \%$ glycerol, $2 \%$ fructose, and $2 \%$ mannose. Cells were then grown for $1 \mathrm{~h}$ at $30^{\circ} \mathrm{C}$. To check compensation mechanisms between CaHXK2 and CaGLK1/4 at the transcriptional level, cells were grown to early log phase on $2 \%$ glucose (YPG). To analyze the influence of the carbon source on CaHxk2GFP and CaGlk1-GFP expression, cells were grown to mid log phase in the different media containing various carbon 
sources. To analyze hexose transporter gene expression, cells were grown to early log phase in lactate $2 \%(\mathrm{OD}=1.8)$ and then transferred to $2 \%$ glucose (YPG). To analyze CaHXK2 and $C a G L K 1 / 4$ transcript level during filamentation assays in liquid medium, cells were grown to $\mathrm{OD}=1.8$ in $0.5 \%$ glucose medium (YP) and then transferred for 30 and $60 \mathrm{~min}$ to $5 \%$ serum. For filamentation assays on solid medium, C. albicans cells were grown for $48 \mathrm{~h}$ at $37^{\circ} \mathrm{C}$ on Spider medium $(1 \%$ Nutrient Broth, $1 \%$ mannitol, $0.2 \% \mathrm{KH}_{2} \mathrm{PO}_{4}, 2 \%$ agar) or $96 \mathrm{~h}$ on YP medium ( $1 \%$ yeast extract, $2 \%$ peptone) supplemented with $2.5 \mathrm{mM}$ GlcNAc. Five percent calf serum was also used to induce the morphological switch at $37^{\circ} \mathrm{C}$ after a $2-5$ days incubation period. The utilization of different carbon sources and sensitivity to different compounds $\left(5 \mathrm{mM} \mathrm{H}_{2} \mathrm{O}_{2}, 1.2 \mathrm{M}\right.$ $\mathrm{KCl}, 0.05 \%$ SDS, $5 \mathrm{mM}$ caffeine) was monitored in liquid YPG at $30^{\circ} \mathrm{C}$ by spectrophotometry (Tecan Infinity 200 Pro Serie). Five $\mathrm{ml}$ of YPG inoculated with stationary phase cells were cultivated to an optical density at $600\left(\mathrm{OD}{ }_{600}\right)=0.6$. Ten $\mu \mathrm{l}$ of culture were used to inoculate $180 \mu \mathrm{l}$ of YP medium containing different carbon sources or the required additives distributed in the wells of a plate. Controls lacking carbon source or specific compounds were performed. Plates were sealed with gas-permeable plastic film. $\mathrm{OD}_{600}$ was measured every $30 \mathrm{~min}$ during $48 \mathrm{~h}$, with shaking at $380 \mathrm{rpm}$. Growth data were based on three independent experiments, each of which consisted of assays performed in triplicate. For growth under hypoxic conditions, aerated flasks were inoculated with an overnight culture of C. albicans, to $\mathrm{OD}_{600}$ of 0.2 . Cells were grown until the beginning of the exponential phase $\mathrm{OD}_{600} \approx 1.8$. Hypoxic conditions were created by collecting and transferring cells suspension in hermetic and filled tubes. Different time points following the shift from normoxic to hypoxic growth conditions were considered (30, 60, 90, and $120 \mathrm{~min})$. After appropriate time, cells were collected by centrifugation at 3,000 rpm for $5 \mathrm{~min}$, washed twice with sterile water and rapidly frozen at $-80^{\circ} \mathrm{C}$. For each time point, three biological replicates were performed. For growth in 96-well plates, anoxic conditions were generated by adding $50 \mu \mathrm{l}$ of mineral oil in each well. Growth values in the presence of stress and hypoxia correspond to the OD values reached after $24 \mathrm{~h}$ of growth for each strain.

\section{Construction of Mutant Strains}

Mutants of the wild type strain SC5314 (Gillum et al., 1984) used in this study are listed in Supplementary Table S1. Mutant strains were constructed using the SAT1 flipper selection cassette kindly provided by J. Morschhäuser (Reuss et al., 2004). CaHXK2 homozygous mutant strain and the complemented strain Cahxk2 $\Delta / \Delta c / c$ were generated according to methods described by Reuss et al. (2004). The Caglk1 $\Delta / \Delta$ and Caglk1glk $4 \Delta / \Delta$ homozygous null mutant strains were constructed by one step cloning-free fusion PCR-based strategy. The Cahxk2glk1 $\Delta / \Delta$ mutant strain was constructed by deleting successively both CaHXK2 alleles of the Caglk1 $\Delta / \Delta$ mutant using the CaHXK2 deletion cassette (Supplementary Data S1). CaHxk2 and CaGlk1 GFP epitope tagging was performed using a PCR-based strategy using pGFP-NAT1 as a template (kindly provided by S. Bates), (Milne et al., 2011). The appropriate mutants were identified by
PCR analysis using a combination of primers outside the sites of cassette integration and internal primers.

\section{Yeast Transformation}

C. albicans transformation was performed using the PEG Lithium technique (Walther and Wendland, 2003). After transformation, mixtures were incubated in YPG for $4 \mathrm{~h}$ at $30^{\circ} \mathrm{C}$ and then plated on YPG + nourseothricin $250 \mathrm{\mu g} \mathrm{ml}^{-1}$ (Werner BioAgent, Jena, Germany). Nourseothricin-sensitive cells were obtained according to Reuss et al. (2004). Transformants were grown overnight in YPG medium without selective pressure. Cells were plated on YPG containing nourseothricin $(25 \mu \mathrm{g} / \mathrm{ml})$. Small colonies containing nourseothricin-sensitive cells were selected after 2 days of growth at $30^{\circ} \mathrm{C}$. Both alleles were disrupted or complemented in a similar manner after elimination of the SAT1 flipper cassette. In the case of in vivo epitope tagging using pGFP-NAT1 (Supplementary Table S2) it was not possible to eliminate the NAT1 marker, one allele was modified by transformation, only.

\section{Yeast Cell Extract and Immunoblotting}

To prepare proteins extracts, cells were suspended in $500 \mu \mathrm{l}$ of 0.1 M Tris-HCl buffer supplemented with $10 \%$ phenylmethylsulfonyl fluoride (PMSF) and then broken in the presence of $1.5 \mathrm{ml}$ of glass beads using a FastPrep ${ }^{\circledR}-24$ (MP Biomedicals) machine (five bursts $6.5 \mathrm{~m} / \mathrm{s}$ for $30 \mathrm{~s}$ ). Following this lysis step, cell extracts were centrifuged at $1,500 \mathrm{rpm}$ for $10 \mathrm{~min}$ at $4^{\circ} \mathrm{C}$. Proteins from the supernatant were quantified using NanoDrop $2000^{\circledR}$.

Immunodetection conditions were as described by Rolland et al. (2006). The $\alpha$-GFP antibody (monoclonal anti-mouse, Roche) and secondary antibody (mouse antibody, HRP conjugated, Bethyl Laboratories) mouse HRP were used at $1 / 5000^{\mathrm{e}}$ and $1 / 10000^{\mathrm{e}}$ final concentration, respectively.

\section{Determination of Hexose Kinase Activity}

Either glucose, fructose, or mannose were used as substrates. The hexokinase II activity was measured spectrophotometrically through $\mathrm{NADP}^{+}$reduction in a glucose-6-phosphate dehydrogenase-coupled reaction. Each reaction was performed in $1 \mathrm{ml}$ spectrophotometer cuvette at room temperature. The final assay mixture consisted of $100 \mu \mathrm{l}$ of $25 \mathrm{mM}$ HEPES buffer $\mathrm{pH} 7.5,100 \mu \mathrm{l}$ of $10 \mathrm{mM} \mathrm{MgCl}_{2}, 100 \mu \mathrm{l}$ of $1 \mathrm{mM} \beta$-NADP, 500 $\mu \mathrm{g}$ of crude proteins extract, two units of glucose-6-phosphate dehydrogenase and (i) $100 \mu \mathrm{l}$ of $10 \mathrm{mM}$ D-glucose for glucose kinase activity, (ii) two units of phosphoglucose isomerase and $100 \mu \mathrm{l}$ of $10 \mathrm{mM} \mathrm{D}$-fructose for fructokinase activity, (iii) two units phosphomannose isomerase, two units of phosphoglucose isomerase, and $100 \mu \mathrm{l}$ of $10 \mathrm{mM} \mathrm{D}$-mannose for mannokinase activity. Reactions were started with the addition of $100 \mu \mathrm{l}$ of $5 \mathrm{mM}$ ATP. Absorbance was continuously recorded at $340 \mathrm{~nm}$, for 10- or 15-min. Activities were obtained from the mean of three independent experiments and expressed as a percentage of the activity obtained with wild type crude protein extract. The apparent $\mathrm{Km}$ of crude extracts of the glucose kinases were determined with a final ATP concentration of $5 \mathrm{mM}$, a final concentration of glucose ranging from $1 \mu \mathrm{M}$ to $100 \mathrm{mM}$. The $\mathrm{NADPH}$ apparition at $340 \mathrm{~nm}$ was measured using a Tecan 
Infinite M200 (Salzburg, Austria) microtiter plate reader at $30^{\circ} \mathrm{C}$. A single well is composed of $10 \mu$ l glucose-6-P dehydrogenase $(0.2 \mathrm{U} / \mathrm{ml}), 10 \mu \mathrm{l}$ of $1 \mathrm{mM} \mathrm{NADP}+, 10 \mu \mathrm{l}$ of $10 \mathrm{mM} \mathrm{MgCl}_{2}$, $10 \mu \mathrm{l}$ of HEPES buffer $(25 \mathrm{mM}, \mathrm{pH} 7.6)$ and $50 \mu \mathrm{g}$ of crude extracts. The reaction was initiated by the addition of $10 \mu \mathrm{l}$ of ATP ( $5 \mathrm{mM}$ in potassium HEPES buffer). The activity was determined using a calibration curve of $\mathrm{NADH}$ in the range of $0-500 \mu \mathrm{M}$ to consider the variability of the optical pathway. The parameters were obtained using Dynafit software and the rapid equilibrium approximation of the Michaelis-Menten equation (Kuzmic, 1996).

\section{GFP Detection by Microscopy}

Yeast strain expressing the CaHxk2::GFP and CaGlk1::GFP fusion proteins were grown to exponential phase $\left(\mathrm{OD}_{600} \approx 0.8\right)$ in YP containing $0.05,0.1$, or $2 \%$ glucose or $2 \%$ lactate. Nuclei were stained by addition of DAPI to $10 \mu \mathrm{g} / \mathrm{ml}$ to the cultures and incubated at $28^{\circ} \mathrm{C}, 180 \mathrm{rpm}$ for $60 \mathrm{~min}$. Cells were washed twice with phosphate buffer saline (PBS) (10 mM Na $2 \mathrm{HPO}_{4}$, $1.76 \mathrm{mM} \mathrm{KH}_{2} \mathrm{PO}_{4}, 137 \mathrm{mM} \mathrm{NaCl}$, and $2.7 \mathrm{mM} \mathrm{KCl}$ ), collected by centrifugation and resuspended in $20 \mu \mathrm{l}$ of PBS. GFP and DAPI localization were monitored in live cells cultures using a Zeiss Axioskop 2 Plus fluorescence microscope. Images were taken with a Zeiss AxioCam MR camera using AxioVision software and processed using LiveQuartz Images Editor.

\section{RNA Extraction and RT-q-PCR Analysis}

Total RNA was extracted from cells grown to $\mathrm{OD}_{600} \approx 1.5$, by the acid phenol method (Collart and Oliviero, 2001). For reverse transcription-quantitative PCR (RT-qPCR) experiments, $10 \mu \mathrm{g}$ of total RNA extract were treated with DNase I (Ambion). Then, ReVertAid H Minus reverse transcriptase (Thermo Scientific), was used as described by the manufacturer, to generate cDNAs. RT-qPCR experiments were performed with the CFX 96 Bio-Rad light cycler using SsoAdvanced Universal SYBR Green Supermix (Bio-Rad). Relative quantification was based on the $2 \triangle C T$ method using CaACT1 (actin) as calibrator. The amplification reaction conditions were as follows: $95^{\circ} \mathrm{C}$ for $1 \mathrm{~min}, 40$ cycles of $95^{\circ} \mathrm{C}$ for $15 \mathrm{~s}, 60^{\circ} \mathrm{C}$ for $30 \mathrm{~s}$, and the final step $95^{\circ} \mathrm{C}$ for $10 \mathrm{~s}$. A melting curve was generated at $95^{\circ}$ for $10 \mathrm{~s}, 65^{\circ} \mathrm{C}$ for $5 \mathrm{~s}$ with an increment of $0.5^{\circ} \mathrm{C}$ until $95^{\circ} \mathrm{C}$ at the end of each PCR cycle, to verify that a specific product was amplified. Primers used in this study are presented in Supplementary Table S4.

\section{Infection of G. mellonella Larvae}

For G. mellonella infection, overnight cultures of WT (SC5314), mutant or complemented strains of $C$. albicans were grown to stationary phase $\left(\mathrm{OD}_{600}=5\right)$ in $2 \%$ YPG medium. Cells were centrifuged and washed three times with $0.9 \% \mathrm{NaCl}$. Larvae were infected with $10 \mu \mathrm{l}$ of suspension $\left(2.5 \times 10^{5}\right.$ cells $)$ injected using a Hamilton syringe, between the third pair of prothoracic legs. Three replicates, each consisting of 10 insects, were carried out with survival rates measured daily for a period of 8 days. Infected larvae were incubated at $37^{\circ} \mathrm{C}$ in the dark. A control group injected with $10 \mu \mathrm{l}$ of $0.9 \% \mathrm{NaCl}$ was included. Death was determined based on the lack of motility and melanisation. Survival curves were plotted and their statistical significance were determined using the GraphPad Prism 7.0 program. $P$-values were estimated using Log rank tests. Surviving larvae were killed at $-20^{\circ} \mathrm{C}$ after experiments following ice-anesthesia.

\section{Infection of Phagocytes With Yeasts}

Macrophages from the J774A.1 (ATCC TIB-67) murine cell line were infected as previously described (Dementhon et al., 2012) in CRPMI medium (RPMI-1640 without phenol red and supplemented with $10 \%$ heat-inactivated fetal bovin serum, $1 \mathrm{mM}$ sodium pyruvate and $2 \mathrm{~g} / \mathrm{l}$ sodium bicarbonate) at $37^{\circ} \mathrm{C}$ under $5 \% \mathrm{CO}_{2}$. Briefly, $2 \times 10^{5}$ macrophages per well were adhered overnight in 96-well plates, and infected with $1 \times 10^{6}$ Calcofluor White (CFW)-labeled yeast cells in stationary phase in cRPMI medium supplemented with $5 \mu \mathrm{g} / \mathrm{ml} \mathrm{CFW}$. Interaction was followed over a 24 -h time course experiment. To count yeasts after phagocytosis, macrophages were infected with C. albicans strains as described in "Materials and Methods" section, using 10 macrophages to 1 yeast multiplicity of infection (MOI). After $4 \mathrm{~h}$ of interaction, the infected macrophages were collected after trypsin treatment, centrifuged for $10 \mathrm{~min}$ at $10,000 \times \mathrm{g}$, and lysed in $1 \mathrm{ml}$ of $0.2 \%$ ice-cold Triton X-100. Released yeast cells were resuspended in YPD and counted using Kova slides (Kova International). Triplicates were done for each experiment. The results are shown as the average of five independent experiments \pm standard errors.

\section{Flow Cytometry Analysis}

Flow cytometry assays were conducted as previously described (Dementhon et al., 2012) using a FACSCantoII (Becton Dickinson). Macrophage viability and the ratio of macrophages engaged in phagocytosis were determined after $30 \mathrm{~min}, 4 \mathrm{~h}$ and $24 \mathrm{~h}$ of infection with CFW-labeled yeasts. Quintuplets of each condition were done for each experiment. After trypsin treatment, macrophages were labeled with $0.2 \mu \mathrm{g} / \mathrm{ml}$ anti-mouse CD16-APC (a membrane stain) and $0.2 \mu \mathrm{M}$ calcein-AM (Sigma) (a marker of active metabolism). The percentage of macrophage viability was calculated using the number of macrophages positive for both fluorescence (anti-CD16-APC and calcein-AM) when infected with yeasts compared to the control uninfected macrophages. Phagocytozing macrophages were quantified as the percentage of the double-stained macrophages also positive for CFW fluorescence. T-test was used to establish statistical significance with a significance level set at $P<0.05$. Gating strategy was based on the specificity of each marker for a cell type. CFW stained yeast cells, calcein and anti-CD16-APC stained macrophages. The threshold for each fluorescence was determined based on positive and negative controls. Yeast without macrophages, in the presence of CFW, were used as positive control for CFW fluorescence. Macrophages alone in the presence of CFW were used as negative control. Cells in absence of CFW also served as negative controls. Yeast and hyphae were identified as positive for CFW fluorescence. Alive macrophages were first identified based on their double fluorescence for calcein (marker for an active metabolism) and anti-CD16APC (membrane marker) (P5 population, Supplementary Figure S3). Then, this P5 population was analyzed for the CFW fluorescence: the triple fluorescent cells (Q2 quadrant, 
positive for calcein, anti-CD16-APC and CFW) are macrophages associated to yeast cells, called "phagocytozing macrophages." Non-phagocytozing macrophages are the macrophages negative for CFW fluorescence (Q4 quadrant).

\section{Statistical Analysis}

All experiments were performed at least three times independently. All statistical data were calculated with GraphPad Prism 7.0 software. For comparisons of multiple groups one-way ANOVA method was used. Significance of mean comparison is annotated as follow: ns, not significant; ${ }^{*} P=0.033$; ${ }^{* *} P=0.002$; ${ }^{* * *} P=0.0002 ;{ }^{* * *} P<0.0001$.

\section{Ethics Statement}

Our study was exempt from ethical approval. The C. albicans SC5314 strain used in this study is referenced in the ATCC collection as ATCCMYA-2876. Murine macrophages used for virulence tests were obtained from the J774A.1 cell line, referenced as ATCC TIB-67 in the ATCC collection. Insect larvae of Galleria mellonella were purchased from Sud-Est Appats ${ }^{1}$ (Quiege, France). There are no legal or ethical restrictions associated with use of Galleria larvae².

\section{RESULTS}

\section{Deciphering the Hexose Kinase Activity in C. albicans}

One hypothetical hexokinase (CaHXK2, GenBank XM_712312) and two hypothetical glucokinase genes (CaGlK1, GenBank XM_705084 and CaGLK4, GenBank XM_707231) were found in the genomic sequence of C. albicans ${ }^{3}$. Analysis of the C. albicans HXK2, GLK1 and GLK4 sequences revealed the presence of a classical hexose kinase conserved domain organized in two regions $s^{4}$ a small and a large subdomain. The small subdomain contains the sugar-binding site of typical hexose kinases: -LGFTFSF/YP- (Bernardo et al., 2007). Protein function prediction ${ }^{5}$ revealed that $\mathrm{CaHxk} 2, \mathrm{CaGlk1}$ and CaGlk4 may be involved in phosphate-containing compound metabolic processes, transferase activity and ATP-binding. The localization of these conserved domains is provided in Supplementary Table S3 (Bork et al., 1992; Kuser et al., 2000). Two nuclear localization sequences were identified in the hexokinase sequence: -PAQKRKGTFT- (8-17) and -QKRGYKTAH- (405-413). These sequences were not found in the glucokinase sequences. Both glucokinase and hexokinase genes are located on chromosome R (Figure 1A), spaced by $70 \mathrm{Kbp}$ and oriented in opposite directions. The CaGLK1 and CaGLK4 sequences share $98.6 \%$ and $99.2 \%$ identity at the nucleotide and amino acid level, respectively. Alignment of the CaGLK1 and CaGLK4 genomic regions showed that a high level

\footnotetext{
${ }^{1}$ http://www.sudestappats.fr/

${ }^{2}$ https://www.legifrance.gouv.fr/eli/decret/2013/2/1/AGRG1231951D/jo/texte

${ }^{3} \mathrm{http}: / /$ www.candidagenome.org/

${ }^{4} \mathrm{http} / / /$ prosite.expasy.org/

${ }^{5} \mathrm{http}: / /$ bioinf.cs.ucl.ac.uk
}

of identity (98\%) spanned from 1,500 bp before and after the coding sequences. Within these conserved regions, separated by a few hundred of base pairs, both glucokinase genes are framed by two uncharacterized coding sequences. Alignment of these sequences, spanning the $5^{\prime}$ and $3^{\prime}$ regions of both glucokinases, revealed a level of $95.1 \%$ and $98.8 \%$ identity, respectively. This strongly indicates that the whole conserved region containing the CaGLK1 and CaGLK4 genes has been duplicated and conserved.

To identify the function of CaHXK2, CaGLK1, and CaGLK4, a set of gene disruption strains was constructed in the C. albicans wild type strain SC5314. Single homozygous null Cahxk2 $($ Cahxk2 $\Delta / \Delta)$ and Caglk1 $($ Caglk1 $\Delta / \Delta)$ mutants and double homozygous null CaHXK2 CaGLK1 (Cahxk2glk1 $\Delta / \Delta)$ and

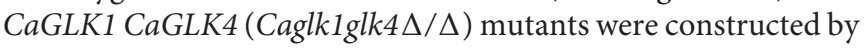
replacing both wild type alleles using the excisable CaSAT1 flipper cassette (Reuss et al., 2004). A CaHXK2 complemented strain $(\operatorname{Cah} x k 2 \Delta / \Delta c / c)$ was also constructed by reintegrating the wild type coding sequence at the HXK2 locus, using the same strategy (Supplementary Data S1).

To investigate the contribution of CaHxk2, CaGlk1, and $\mathrm{CaGlk} 4$ to the phosphorylation of hexoses in C. albicans, we measured the hexose kinase activity displayed by the wild type strain (SC5314) and the generated mutant strains (Figure 1B). Data obtained with Cahxk2 $\Delta / \Delta$ cell extracts revealed that glucose kinase and mannose kinase activities decreased by $65 \%$ and $75 \%$, respectively, while the phosphorylation of fructose was totally abolished. This suggests that other enzymes, like glucokinases, could phosphorylate glucose and mannose, while fructose is phosphorylated by $\mathrm{CaHxk} 2$ only. Values obtained with the complemented strain Cahxk $2 \Delta / \Delta c / c$, statistically comparable to the data from the wild type strain, indicated that the lack of fructose phosphorylation was due to the deletion of the gene. Deletion of one or both glucokinase genes (CaGLK1, CaGLK4) had no apparent consequence on the level of hexose phosphorylation, suggesting that glucokinase activity could be compensated by the hexokinase activity. Hexose kinase activity measured in the Cahxk2 $\Delta / \Delta$ strain corresponds to the sum of the activities of $\mathrm{CaGlk1}$ and CaGlk4 (33\% of the total activity). Glucose kinase activity, measured in the double mutant strain Cahxk2glk1 $\Delta / \Delta$, which corresponds to the activity of CaGlk4, was drastically reduced compared to the Cahxk $2 \Delta / \Delta$ mutant. This activity corresponds to $6 \%$ of the total glucose kinase activity. Taken together these results suggest that glucokinases enzymes contribute unevenly and seem to play a minor role in glucose phosphorylation in the wild type strain.

To further investigate the specificity of $\mathrm{CaHxk} 2, \mathrm{CaGlk1}$, and $\mathrm{CaGlk} 4$, we determined their apparent Michaelis constant for glucose (Figure 1C). Apparent $K_{\mathrm{m}}$ of $\mathrm{CaHxk} 2$ was measured in the Caglk1glk $4 \Delta / \Delta$ strain, while the $K_{\mathrm{m}}$ of Glk4 was determined in Cahxk2glk1 $\Delta / \Delta$ strain. The apparent $K_{\mathrm{m}}$ of Glk1 was estimated in the Cahxk2 $\Delta / \Delta$ by subtracting the effect of CaGlk4. Data revealed that hexokinase 2 and glucokinase 1 have much lower $K_{\mathrm{m}}$ values $\left(K_{\mathrm{m}} 104.87 \pm 7.05 \mu \mathrm{M}\right.$ and $K_{\mathrm{m}} 84.86 \pm 6.23$ $\mu \mathrm{M}$, respectively) than glucokinase $4\left(K_{\mathrm{m}} 3900 \pm 400 \mu \mathrm{M}\right)$, using glucose as a substrate. The low glucose affinity of $\mathrm{CaGlk} 4$ could partially explain the poor contribution of this protein to glucose and mannose phosphorylation. 

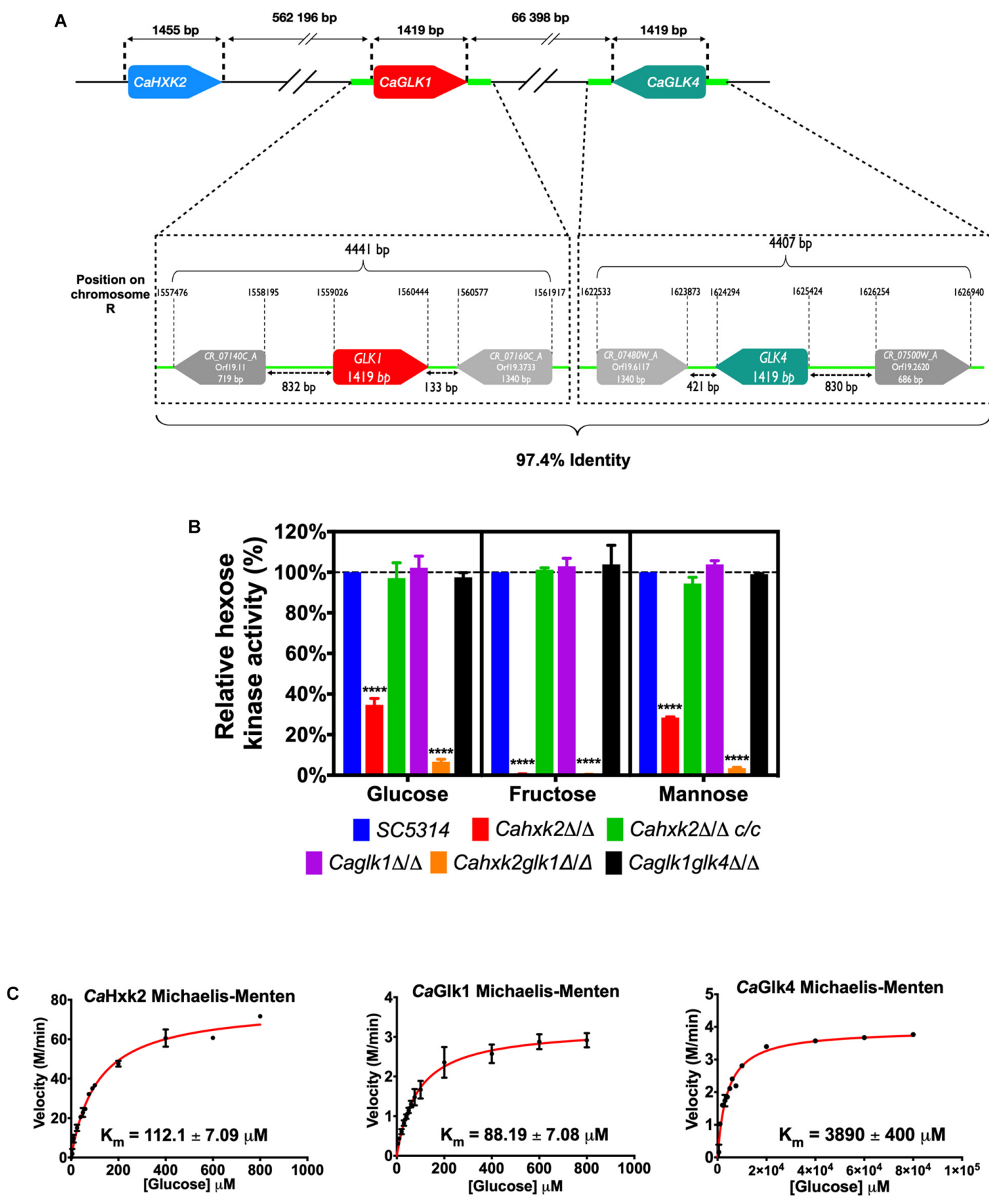

FIGURE 1 | The hexose kinase system in C. albicans. (A) The hexose kinase genes are located on C. albicans chromosome RA (Ca22chrRA_C_albicans_SC5314:994,376. . 997,830). Glucokinase genes are oriented in opposite directions and bordered by highly homologous regions (light green). (B) Hexose phosphorylation rates in C. albicans wild type and hexose kinase mutant cell extracts. For each strain, the amount of glucose-6-phosphate produced was measured and expressed as a percentage of the wild type strain. Data are presented as a mean (+ standard deviation) of three independent experiments performed on three different biological samples, in triplicates ( $n=9$ ); ${ }^{* * * *} P<0.0001$; one-way ANOVA using Dunnett's method. (C) Kinetic constant of hexose kinases (apparent $K_{m}$ determined with crude extracts) in C. albicans in the presence of glucose. The experiment was performed in triplicate. Representative data are presented here.

\section{Hxk2 Mostly Sustains Growth in the Presence of Hexoses}

Impact of hexokinase and glucokinase gene deletion on growth in the presence of hexoses was evaluated (Figure 2). Delayed growth of the hexokinase mutant $\operatorname{Cah} x k 2 \Delta / \Delta$ on glucose and mannose and severely impaired growth on fructose, confirmed the absence of a functional hexokinase. Slow growth on glucose and mannose was consistent with the presence of an additional glucokinase 

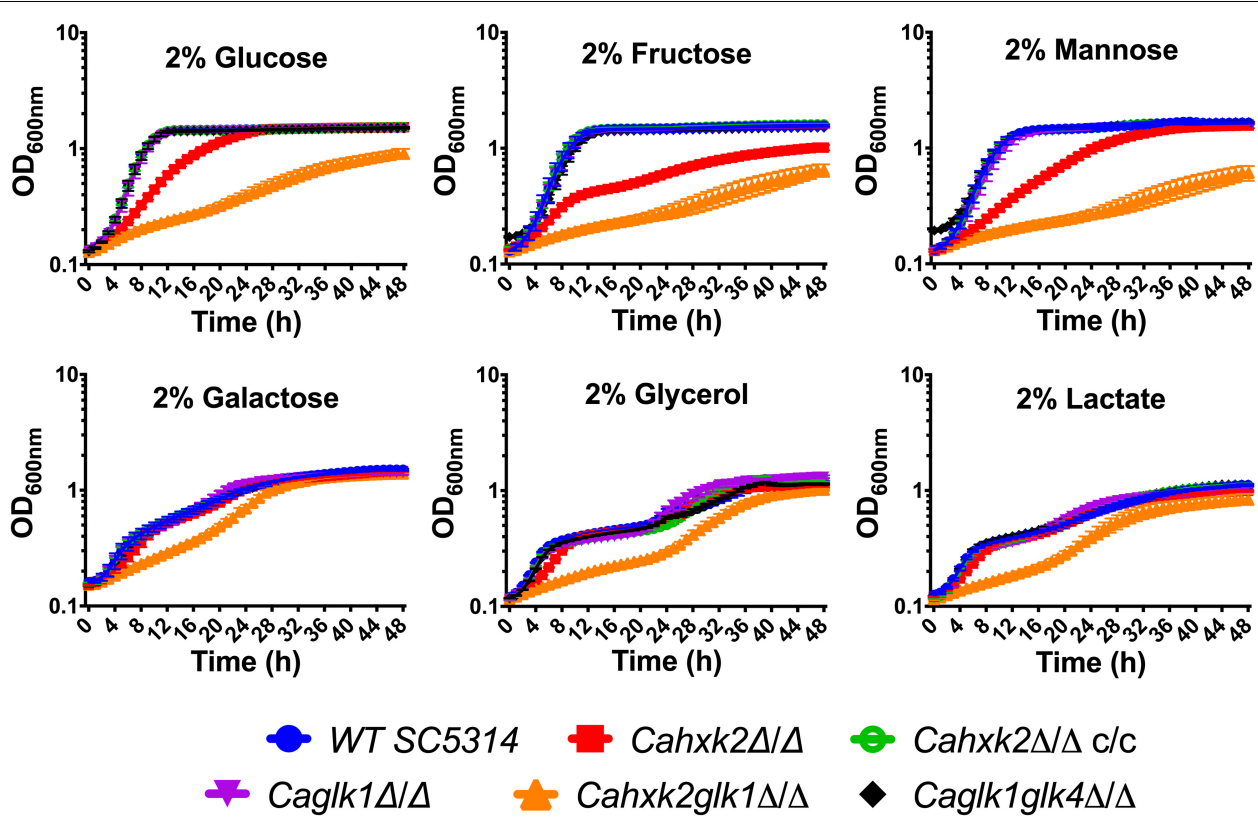

FIGURE 2 | Hexokinase 2 is necessary to sustain C. albicans growth. Five $\mathrm{ml}$ of YPG inoculated with stationary phase cells were cultivated to OD $600=0.6$. Ten $\mu$ l of culture were used to inoculate $180 \mu \mathrm{l}$ of YP medium containing different carbon sources. A 96-well plate containing appropriate medium was inoculated with each strain at starting $\mathrm{OD}_{600}=0.2$. Cells growth was performed at $30^{\circ} \mathrm{C}$ and recorded during $48 \mathrm{~h}$ using microplates reader (TECAN infinite pro200). Data are presented as a mean ( \pm standard deviation) of three independent experiments performed on three different biological samples, in triplicates $(n=9)$.

activity. The strong growth defect observed on fructose for this mutant confirmed the fact that fructose is phosphorylated by $\mathrm{CaHxk} 2$ only. The residual growth observed on fructose could be due to the metabolism of the alternative carbon sources present in YPG. Growth of the mutant Cahxk2 $\Delta / \Delta$ was not affected in the presence of glycerol or galactose, substrates that are not phosphorylated by $\mathrm{CaHxk} 2$. This indicates that growth defects are linked to an impaired phosphorylation of hexoses. Moreover, growth of the complemented strain was comparable to the wild type. Altogether, these data clearly show that the hexokinase $\mathrm{CaHxk} 2$ is necessary for proper growth in C. albicans in the presence of glucose, fructose and mannose.

Deletion of CaGLK1 or both CaGLK1 and CaGKLK4 did not affect growth. Growth of the double mutant Cahxk2glk1 $\Delta / \Delta$ was drastically affected in the presence of glucose, fructose and mannose. Growth failure was also observed, but less pronounced, in the presence of carbon sources that are not phosphorylated by hexokinase or glucokinase (galactose, lactate, glycerol). This strongly suggests that the presence of CaGlk4 alone is not sufficient to sustain growth in the presence of hexoses and that the lack of both $\mathrm{CaHxk} 2$ and $\mathrm{CaGlk} 1$ could affect general physiological properties, beyond hexose phosphorylation in C. albicans.

\section{Glucokinases and Hexokinase Do Not Compensate at the Transcriptional Level and Are Differentially Regulated}

To highlight the respective role of hexokinase and glucokinases, CaHXK2, CaGLK1, and CaGLK4 expression was analyzed.
To check the influence of the carbon source, cells were grown on $2 \%$ lactate and transferred to different media (Figures 3A,B). Due to the high level of homology of their coding sequences $(98.6 \%$ identity) it was not possible to amplify CaGLK1 transcripts alone. Therefore, the transcription level corresponded to the sum of CaGLK1 and CaGLK4 transcripts (indicated as CaGLK1/4). In the presence of glucose, CaHXK2 was three times more expressed than CaGLK1/4 (Figure 3A). Transcription of hexokinase and glucokinase genes was strongly induced by glucose $(0.1 \%$ and $2 \%)$. In the presence of glucose, CaHXK2 was three to five times more expressed that CaGLK1/4 (Figures 3A,B). Contrary to glucokinase genes, the level of CaHXK2 transcripts was dependent on the glucose concentration. Transcription of hexose kinase genes was also strongly induced by mannose and fructose. Surprisingly, the transcription of glucokinase genes was induced by fructose and glycerol which are not substrates for glucokinases (Figure 3B).

To better elucidate hexose kinase gene regulation, we examined their transcription after growth on $2 \%$ glucose in the different mutant strains (Figure 3C). Expression data confirmed an absence of transcripts in the corresponding genedeleted strains and revealed a complete restoration of the hexokinase transcription level after re-introduction of both wild type alleles. CaHXK2 expression was not increased in the glucokinase mutants (Caglk1 $\Delta / \Delta$, Caglk1glk $4 \Delta / \Delta)$. Likewise,

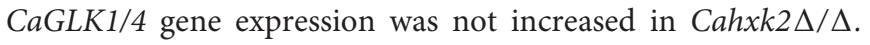
This suggests that unlike what happens in S. cerevisiae (Moreno and Herrero, 2002), no compensation mechanisms interfere to regulate glucokinases and hexokinase at the transcriptional level in the absence of one or the other gene. This points out different 
A

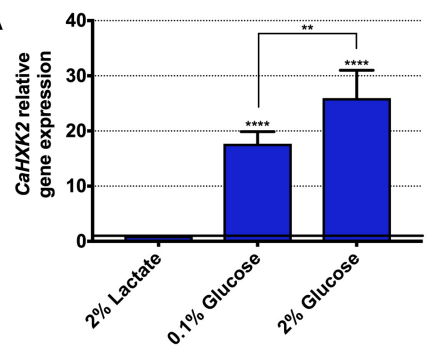

B

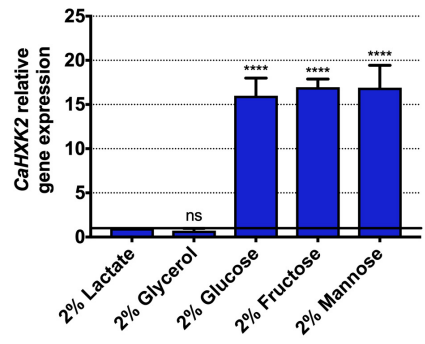

C

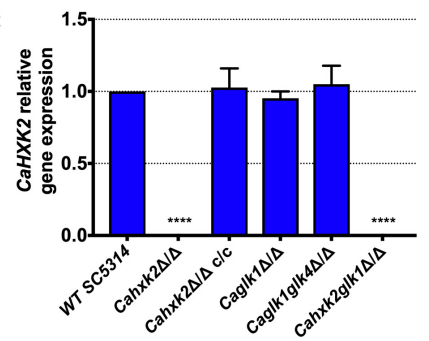

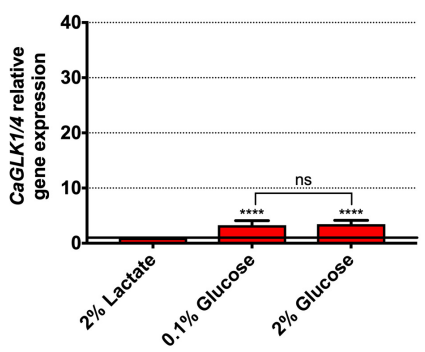

D

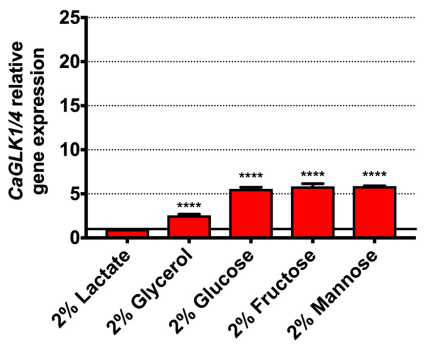

F

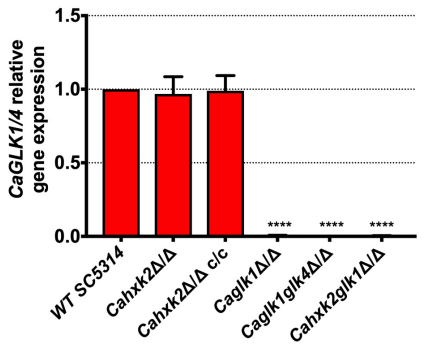

E
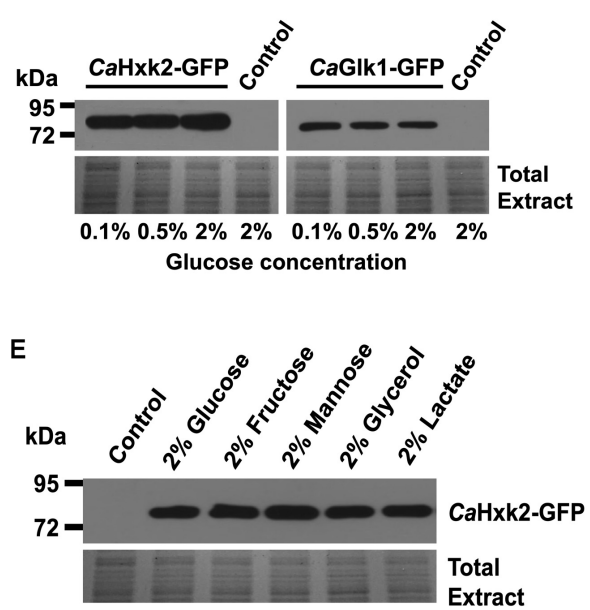

Glucose concentration

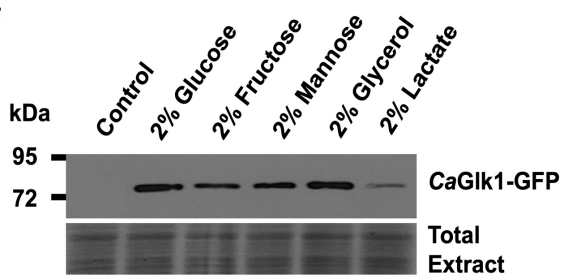

FIGURE 3 | Hexokinase and glucokinases expression. (A) Relative expression of CaHXK2 and CaGLK1/4 in wild type strain in presence of $2 \%$ lactate, $0.1 \%$ and $2 \%$ glucose. Lactate-grown cells (OD = 1.8) were transferred for $1 \mathrm{~h}$ to different media containing $2 \%$ carbon sources (B) Relative expression of CaHXK2 and CaGLK1/4 in wild type strain in presence of various carbon sources (2\% lactate, glycerol, glucose, fructose or mannose). For the panels (A,B) the results were normalized to the expression of CaACT1. The level of CaHXK2 and CaGLK1/4 mRNAs was expressed relatively to their abundance in $2 \%$ lactate, which was set to 1. Lactate-grown cells $(O D=1.8)$ were transferred for $1 \mathrm{~h}$ to different media containing $2 \%$ carbon sources (C) Relative expression of CaHXK2 and CaGLK1/4 in wild type and hexose kinase mutant strains after growth in $2 \%$ YPG $(O D=1.8)$. The expression was normalized to the level of the CaACT1 mRNA internal control. mRNA levels were expressed relatively to their abundance in the wild type strain, which was set to 1. Results represent a mean (+ standard deviation) of three independent experiments performed on three different biological samples, in duplicate $(n=6)$; ns, non-significant; ** $P=0.002$; **** $P<0.0001$. $P$-values were calculated by one-way ANOVA using Tukey's method. (D) Strains of C. albicans expressing C-terminally GFP-tagged CaHxk2 or CaGlk1 were cultivated in presence of 0.1, 0.5 , or $2 \%$ glucose to mid log phase. Whole cell lysates were analyzed for CaHxk2-GFP and CaGlk1-GFP by western blotting, using $\alpha$-GFP antibody. Detection of total proteins by in-gel Coomassie staining was used as a loading control (total extract). Western blots were performed three times. Representative data are presented here. (E,F) C. albicans expressing C-terminally GFP-tagged CaHxk2 or CaGlk1 were cultivated in presence of various carbon sources (2\%) to mid log phase. Whole cell lysates were analyzed for CaHxk2-GFP and CaGlk1-GFP by western blotting, using $\alpha$-GFP antibody. Wild type strain protein extracts were loaded as control. Detection of total proteins by in-gel Coomassie staining was used as a loading control (total extract). Western blots were performed three times. Representative data are presented here.

regulation pathways. However, this compensation could occur at the protein level since the double glucokinase mutant shows no hexose phosphorylation deficiency. Moreover, the fact that the level of $C a G L K 1 / 4$ transcripts was unchanged in the absence of the hexokinase $(\operatorname{Cah} x k 2 \Delta / \Delta)$ revealed that glucokinases genes are not subjected to glucose repression (Figure 3C). Interestingly, the level of CaGLK4 expression detected in Caglk1 $\Delta / \Delta$ and $C a h x k 2 g l k 1 \Delta / \Delta$ was very low, just above the detection threshold. Considering that the glucokinase gene expression level detected in the mutant $\operatorname{Cah} x k 2 \Delta / \Delta$ is the sum of GLK1 and GLK4 transcripts, we can again assume that $C a G L K 1$ and $C a G L K 4$ are not equally expressed.

To investigate the expression of the enzymes, we constructed HXK2::GFP and GLK1::GFP strains (CaHXK2-GFP and
CaGLK1-GFP) expressing CaHXK2-GFP and CaGLK1-GFP from their own promoters. GFP-tagged CaHxk2 and CaGlk1 were detected in cell extracts by immunoblotting, after growth in the presence of glucose. CaHxk2-GFP and CaGlk1-GFP were detected whatever the glucose concentration. However, CaHxk2-GFP was much more abundant than CaGlk1-GFP (Figures 3D-F). This is consistent with the higher transcription level observed for CaHXK2 but could also reflect a faster turnover for glucokinases. CaHxk2-GFP was equally detected in the presence of various carbon sources that are inducers of its transcription and substrates of the enzyme, but also in the presence of glycerol and lactate that do not induce CaHXK2 transcription (Figure 3B). This could be explained by the long half-life of $\mathrm{CaHxk2}$. In addition to the lowest abundance 
of CaGlk1-GFP, the main difference between CaHxk2-GFP and $\mathrm{CaGlk1-GFP}$ protein content was that $C a G l k 1$ was barely detectable in cell extracts after growth on lactate. This may again

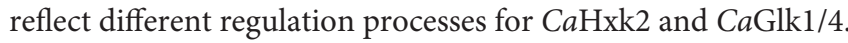

\section{Hexokinase Mediates Glucose Repression but Not Glucokinases}

To highlight the regulatory functions of $\mathrm{CaHxk2}$, we examined the localization of $\mathrm{CaHxk2-GFP}$ in living cells exposed to glucose (Figure 4A). Upon growth in glucose (2\%) the GFP signal was distributed in all the cell (except in the vacuole) with a strong accumulation into a structure that colocalize with the nucleus. This nuclear GFP signal was less apparent in cells grown in $0.1 \%$ glucose and nearly absent in cells grown without glucose (2\% lactate) or at very low glucose concentration $(0.05 \%)$. This indicates that, in C. albicans, CaHxk2 is able to shuttle from the cytoplasm to the nucleus in presence of high glucose $(0.1 \%$ and more). This observation is similar to what observed in S. cerevisiae grown in $2 \%$ glucose where Hxk2 is known to accumulate into the nucleus where it exerts a transcriptional regulatory function necessary for glucose repression independent of its hexokinase activity (Randez-Gil et al., 1998; FernándezGarcía et al., 2012). The cellular localization of the glucokinases was also investigated. Upon growth of CaGlk1-GFP on 2\% glucose, the GFP signal was not detected in the nucleus (Supplementary Figure S1).

To ascertain the impact of CaHxk2, CaGlk1, and CaGlk4 on glucose repression, we analyzed the expression of high affinity hexose transporter genes (Fan et al., 2002) that are known to be controlled by the central repressor of the glucose repression pathway, CaMig1, in response to glucose (Zaragoza et al., 2000; Sexton et al., 2007) (Figure 4B). These transporter genes (CaHGT7, CaHGT12, CaHXT10) are also regulated by another main glucose sensing pathway, the SRR pathway, except CaHGT8 which is not (Brown et al., 2006). Hexose transporter gene expression was drastically enhanced (up to 10 times for CaHXT10) in the hexokinase mutant after transfer on $2 \%$ glucose medium. On the contrary, in the double glucokinase mutant expression level was either lowered in the case of CaHGT7, CaHGT12, and CaHXT10 or unaffected in the case of CaHGT8. These data suggest that CaHxk2 but not glucokinases, could have a repressor function on hexose transporter gene expression. Moreover, SRR-dependent CaHGT7, CaHGT12, CaHXT10 under-expression in the glucokinase mutant may suggest an unexpected regulatory role for glucokinase in transporter gene expression.

\section{Hexose Kinase Enzymes Mediate Protection During Harmful Environmental Challenges: Glucokinase Contributes to the Hypoxic Response}

In C. albicans and number of yeasts, one strategy to counteract oxidative and osmotic stresses is the rapid endogenous synthesis of compatible solutes or, under exposure to cell wall stresses, cell wall biogenesis (Eisman et al., 2006; Sánchez-Fresneda et al., 2013). These stress responses which are directly or indirectly linked to glucose metabolism, could have been affected in the hexose kinase mutants. For that purpose, wild type and mutant strains were grown in the presence of $2 \%$ glucose (YPG) supplemented with $1.2 \mathrm{M} \mathrm{KCl}$ (osmotic stress), $5 \mathrm{mM} \mathrm{H}_{2} \mathrm{O}_{2}$ (oxidative stress), $0.05 \% \mathrm{SDS}$ and $5 \mathrm{mM}$ caffeine (cell wall stresses). Data presented Figure 5A that compare OD values of each strain in the presence and absence of stress after $24 \mathrm{~h}$ of growth in microplates, revealed that all stresses had an impact by decreasing growth of the hexokinase mutants (Cahxk2 $\Delta / \Delta$, Cahxk2glk1 $\Delta / \Delta)$. Conversely, single and double glucokinase mutant strains were not significantly susceptible to the applied stresses. This suggests that Cahxk2 is involved in stress responses through its central metabolic position.

During host infection, C. albicans colonizes multiple niches that greatly differ in oxygen content, meaning that it is adapted to hypoxic environments. Growth of the wild type and mutant strains under hypoxic conditions revealed the impact of CaHXK2 deletion (Figure 5B). Growth of Cahxk2 $\Delta / \Delta$ and Cahxk2glk1 $\Delta / \Delta$ was affected by $50 \%$ after $24 \mathrm{~h}$, as compared to normoxia while the deletion of one or two glucokinases had minor or no effects. The transcriptional response to hypoxia, elucidated in C. albicans, revealed a global upregulation of glycolytic genes (Setiadi et al., 2006; Sellam et al., 2014). This prompted us to investigate the expression of hexokinase and glucokinase in response to hypoxia (Figure 5C). After $1 \mathrm{~h}$ of exposure, CaGLK1/4 transcript level increased by a factor of 25, while CaHXK2 upregulation was much less detectable. This shows that glucokinases and hexokinase transcription is differently regulated by hypoxic conditions. This was confirmed at the protein level. GFP-tagged hexokinase was equivalently detected in normoxia and hypoxia. In contrast, immunoblot of $\mathrm{CaGlk1-GFP}$ revealed a constant amount of protein in response to hypoxia that persisted along the growth, while in normoxia, the amount of $\mathrm{CaGlk1-GFP}$ detected clearly decreased (Figure 5D).

\section{Hexose Phosphorylation by CaHxk2 Is Necessary to Filamentation}

As glucose is one of the several stimuli that can trigger yeast-to-hypha development in C. albicans (Biswas et al., 2007), we checked the ability of hexokinase and glucokinase mutants to undergo a yeast-to-hyphae morphological transition. To evaluate the impact of the hexose phosphorylation step on filamentation, hyphal formation was induced by growth on different media containing known inducing carbon sources, requiring or not hexose kinase enzymes for further metabolization. Spider and serum media, contain, respectively, mannitol and glucose that depends upon the hexose kinase step to be metabolized. The third medium contains $\mathrm{N}$-acetylglucosamine, that does not require Cahxk2, CaGlk1, or CaGlk4 to be metabolized, but require CaHxk1 (Yamada-Okabe et al., 2001; Rao et al., 2013). After 2 days of growth at $37^{\circ} \mathrm{C}$ on serum and spider media, the wild type, CaHXK2 complemented strain and glucokinase mutants showed abundant filaments at the periphery of the colony, while the hexokinase mutants $(\operatorname{Cah} x k 2 \Delta / \Delta$, Cahxk2glk1 $\Delta / \Delta)$ produced hyphae-deficient colonies (Figure 6A). Morphological changes were also observed at the cell level. Microscopic 


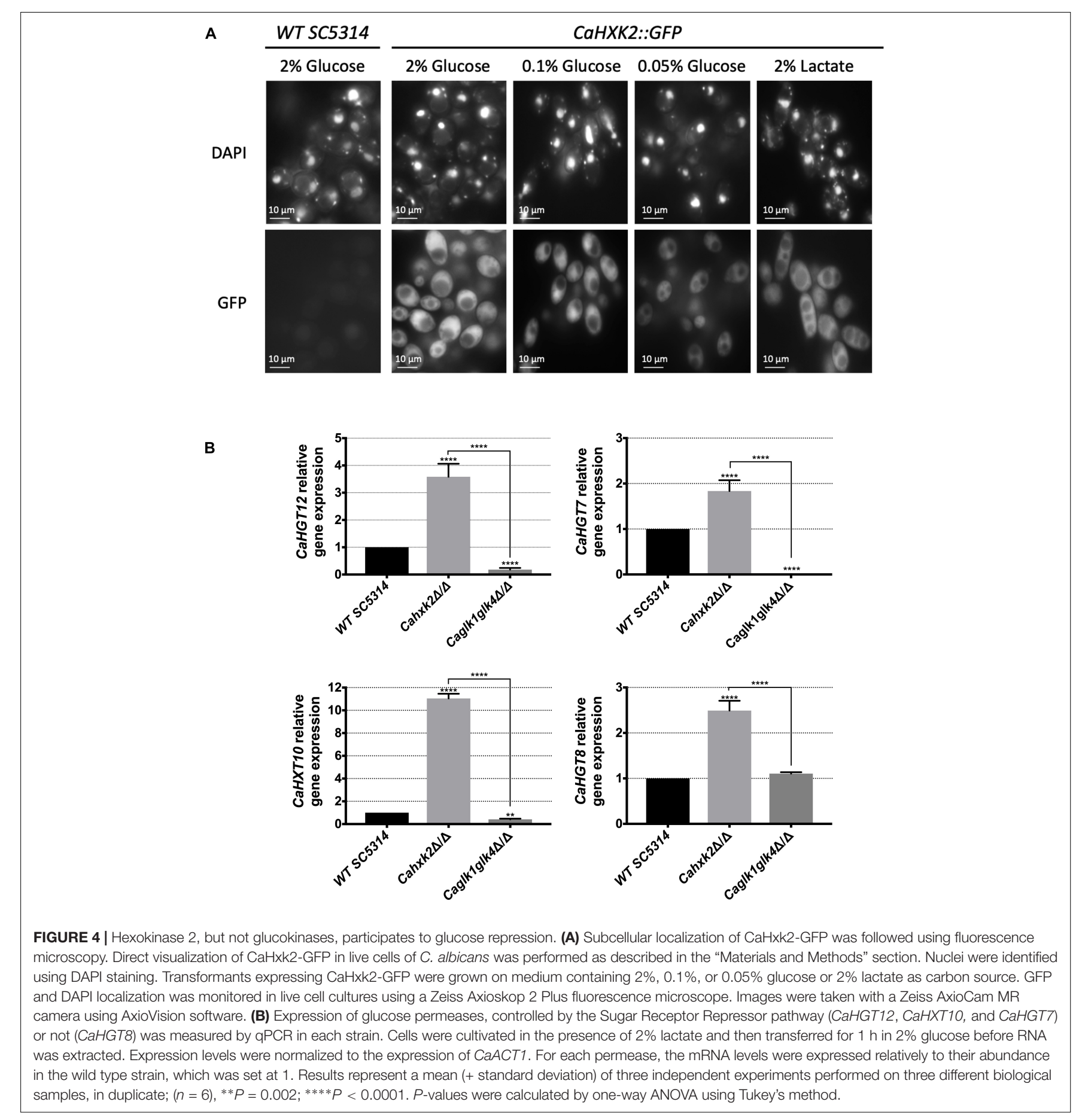

observations revealed a drastically decreased proportion of filamentous structures for the hexose kinase mutant cells, suggesting that the hexokinase CaHXK2 is necessary to the yeast-to-hyphae transition. By contrast, filamentation of the Cahxk2 $\Delta / \Delta$ hexokinase mutant was not affected during growth in the presence of $N$-acetylglucosamine. This suggests that hexose phosphorylation by $\mathrm{CaHxk} 2$ is an essential step for filamentation. The double mutant Cahxk2glk1 $\Delta / \Delta$ behave in a similar way to the $\operatorname{Cah} x k 2 \Delta / \Delta$ hexokinase mutant on spider and serum media but not in the presence of GlcNAc, where it appeared hypofilamentous. This filamentation defect of the double mutant Cahxk $2 g l k 1 \Delta / \Delta$ grown on the particular carbon source GlcNAc, may be the consequence of severe physiological disturbances.

To eventually highlight a specific role in pathogenic behavior for C. albicans hexose kinases, we compared hexokinase and glucokinase gene expression levels during the early steps of the morphological switch. Our data did not reveal any particular transcriptional response of one gene or another (Figure 6B). Both 
A
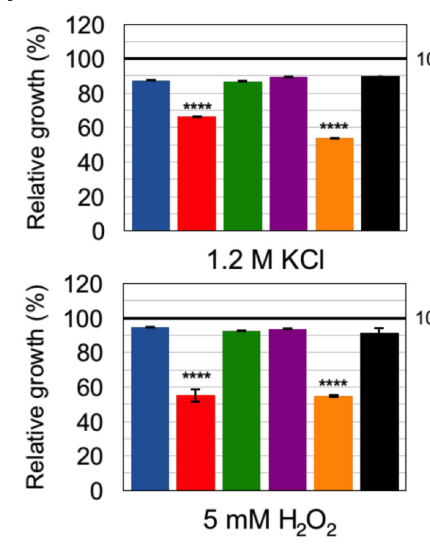

WT SC5314

Caglk1 $1 \Delta / \Delta$

C

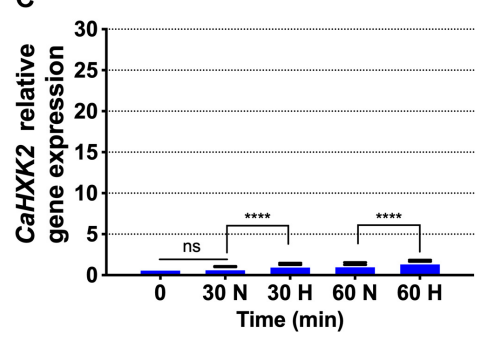

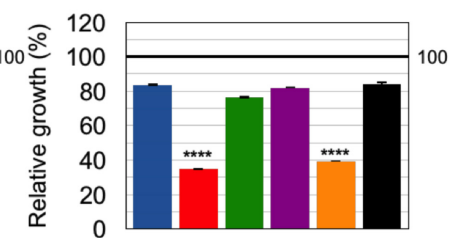

$0.05 \%$ SDS

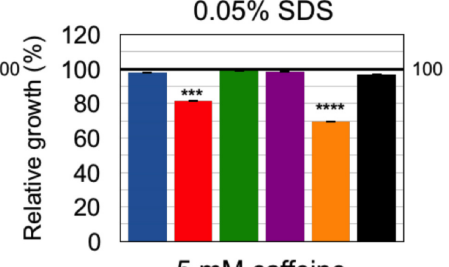

$5 \mathrm{mM}$ caffeine

Cahxk2 $\Delta \Delta \mathrm{c} / \mathrm{c}$

Caglk1glk $4 \Delta / \Delta$

B

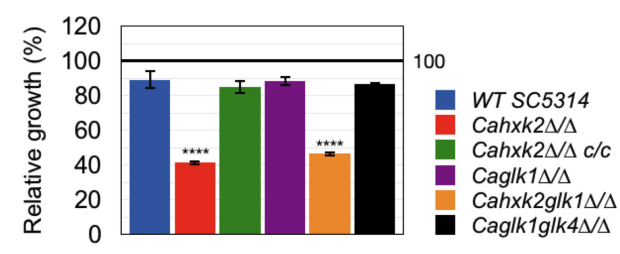

D
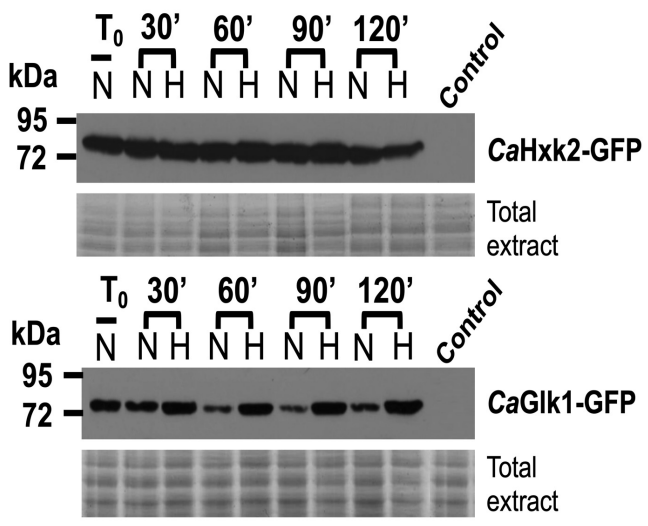

$\mathrm{N}=$ Normoxia $\mathrm{H}=$ Hypoxia

FIGURE 5 | Hexose kinase enzymes mediate protection during harmful environmental challenges. (A) Growth of the wild type and hexose kinase mutant strains exposed to various stresses in YPG was expressed as a percentage of the growth of each strain in absence of stress which was set to 100\% (black line). Data correspond to the OD values reached after $24 \mathrm{~h}$ of growth in microplates. (B) Growth of the wild type and hexose kinase mutant strains under hypoxic conditions in $2 \%$ glucose YPG was expressed as a percentage of growth in normoxia which was set to 100\% (black line). (C) Relative expression of CaHXK2 or CaGLK1/4 measured in normoxia (N) or hypoxia (H) during growth in YPG $2 \%$ glucose. Transcript level was analyzed by qPCR at 0,30 and 60 min. Results were normalized to the CaACT1 transcript level. The level of CaHXK2 and CaGLK1/4 mRNAs was expressed relatively to their abundance at time zero, which was set to 1. Histograms represents a mean of three independent experiments performed on three different biological samples, in triplicate $(n=9)$; ns, non-significant; *** $P=0.0002$; ****P $<0.0001$. $P$-values were calculated by one-way ANOVA using Tukey's method. (D) Strains of $C$. albicans expressing CaHxk2-GFP or CaGlk1-GFP were grown in $2 \%$ glucose YPG to the mid log phase. Cells were transferred into the new medium containing $2 \%$ glucose and exposed ( $\mathrm{N}=\mathrm{Normoxia}$ ) or not ( $\mathrm{H}=$ Hypoxia) to oxygen. Following this shift, cells were collected at 0, 20, 60, 90, and 120 min and the detection of CaHxk2-GFP or CaGlk1-GFP was performed by western blot using $\alpha$-GFP antibody. Wild type strain protein extracts were loaded as control. Detection of total proteins by in-gel Coomassie staining was used as a loading control (total extract). Western blots were performed three times. Representative data are presented here.

profiles revealed a two-time increase of transcripts 30 or $60 \mathrm{~min}$ after the initiation of the filamentation by serum and a shift at $37^{\circ} \mathrm{C}$. However, after $1 \mathrm{~h}$ of growth, glucokinases expression continues to increase while CaHXK2 transcription level decreases after $30 \mathrm{~min}$.

\section{Cahxk2 Mutant Is Hypovirulent in Galleria mellonella and Macrophage Models}

To explore the impact of altering hexokinase and glucokinases on $C$. albicans virulence, we examined first the survival rate of the host model $G$. mellonella following infection with the wild type, mutant and complemented strains (Figure 7A). G. mellonella survival data indicated that there was a statistically significant difference between the survival rate of larvae infected by the mutant and the wild type strains, except for the Caglk $1 \Delta / \Delta$ single mutant. Seven days post infection, $100 \%$ of the larvae were killed by the wild type strain while the survival of the larvae infected by $C a h x k 2 \Delta / \Delta$ and Cahxk2glk1 $/ \Delta$ was still $70 \%$ and $85 \%$, respectively. The double glucokinase mutant was also significantly less virulent than the wild type strain, with an intermediary survival rate of $50 \%$. The Cahxk $2 \Delta / \Delta c / c$ complemented strain revealed a partially restored virulence. These data suggest that strains lacking the hexokinase or both glucokinases show an affected virulence in the G. mellonella model.

Secondly, we analyzed the ability of the mutant to kill macrophages at different interaction times using an in vitro model assay (Figure 7B). Flow cytometer analysis showed that hexokinase and glucokinase gene deletions did not modify macrophages association with yeast for any strains, except for the Cahxk2glk1 $\Delta / \Delta$ double mutant which shows a slightly decreased number of recruited macrophages at the early time of infection 

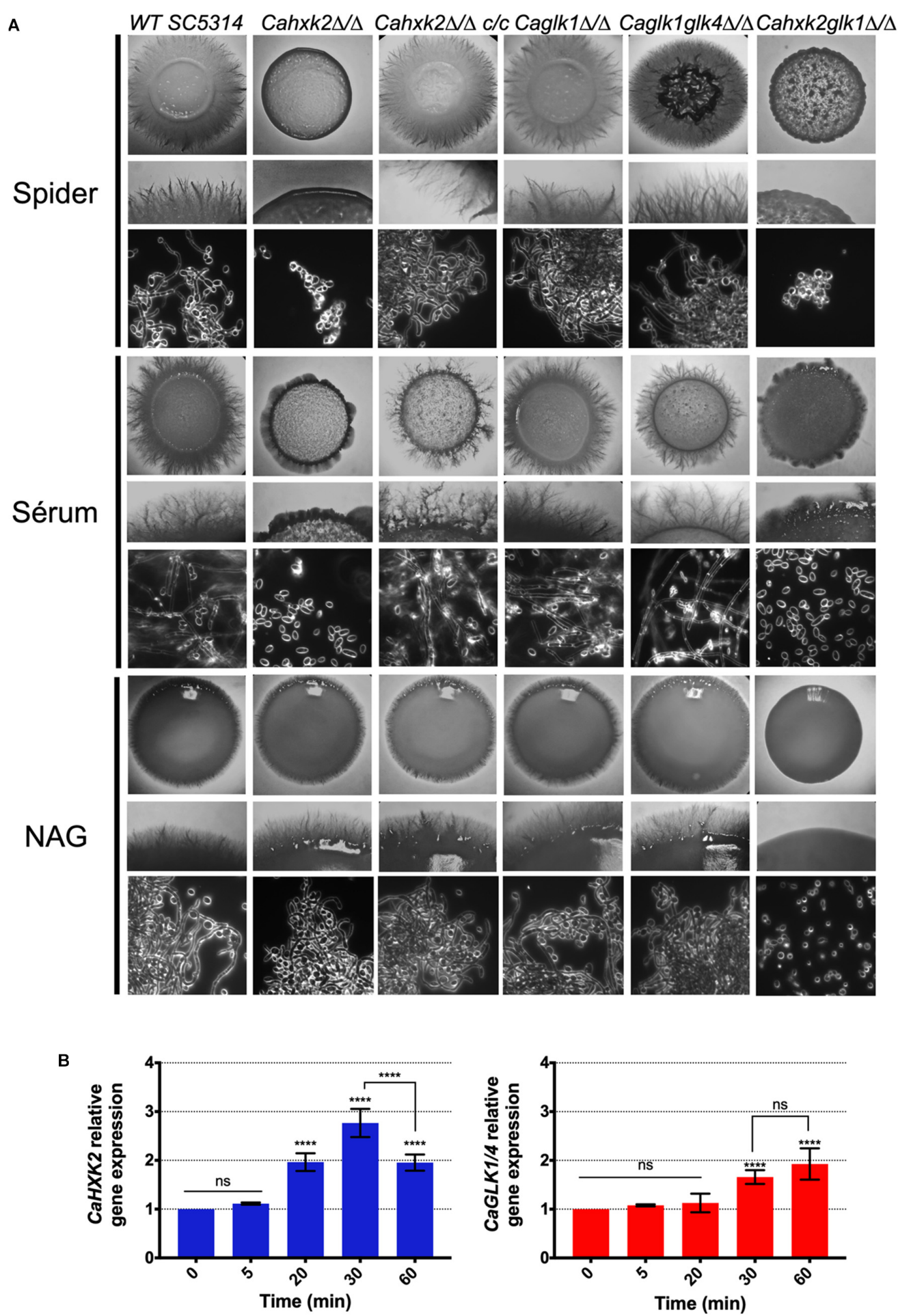

FIGURE 6 | Hexose phosphorylation is required to sustain filamentation. (A) C. albicans wild type and mutant strains were grown during 3 days at $37^{\circ} \mathrm{C}$ on spider, serum or $\mathrm{N}$-acetyl-glucosamine medium. For each medium, the upper and middle panels show photographs of macroscopic appearance of the colonies. Photographs present in the lower panel were obtained using Zeiss Axioskop 2 Plus microscope with dark field and show the microscopic aspect. (B) Relative expression of CaHXK2 and CaGLK1/4 in the wild type strain during filamentation after transfer from $0.5 \%$ YPG to $5 \%$ serum liquid medium. Expression level of CaHXK2 and CaGLK1/4 was measured by qPCR, at different time points (0, 5, 20, 30, and 60 min), and normalized to the level of the CaACT1 mRNA internal control. mRNAs level was expressed relatively to their abundance at time zero, which was set to 1. Results represent a mean (+ standard deviation) of three independent experiments performed on three different biological samples, in duplicate $(n=6)$; ns, non-significant; ${ }^{* * * *} P<0.0001$. $P$-values were calculated by one-way ANOVA using Tukey's method. 
A
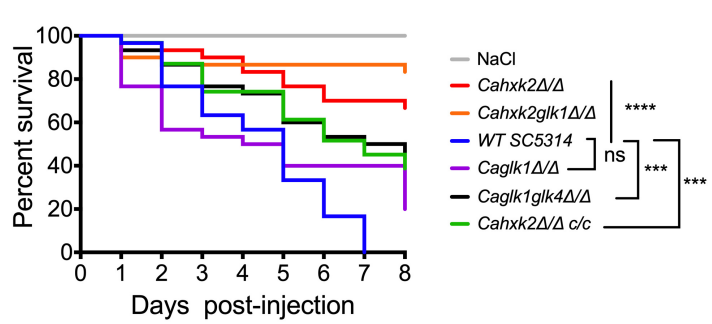

C

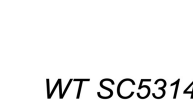

Cahxk2 $\Delta / \Delta$

Cahxk2 $\Delta / \Delta \mathrm{c} / \mathrm{c}$

Caglk1 $1 \Delta / \Delta$

Caglk1glk4 $\Delta / \Delta$

Cahxk2glk1 $1 \Delta / \Delta$
Time post infection

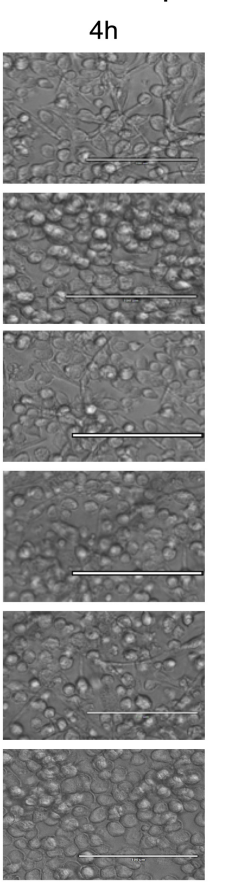

B

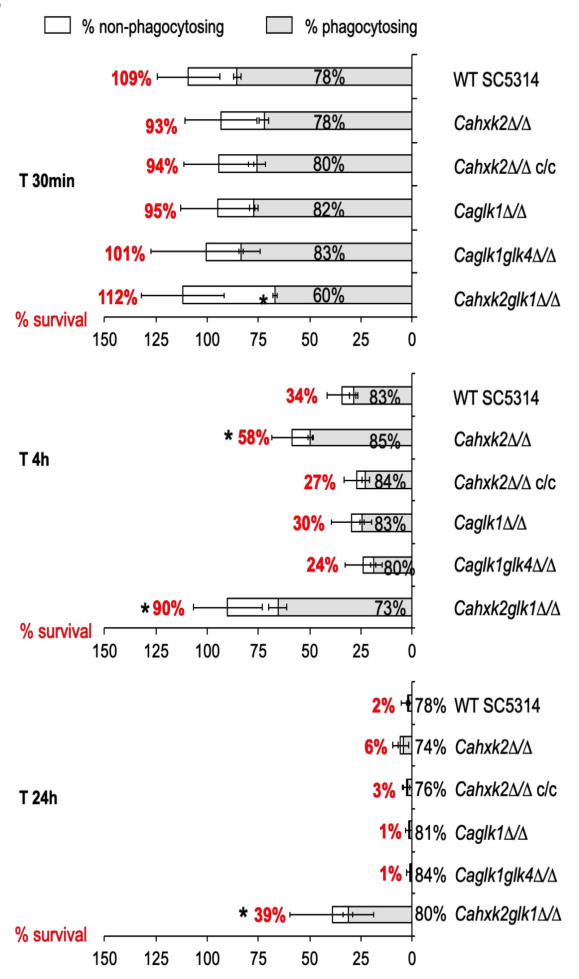

FIGURE 7 | Hexokinase 2 is required for full virulence of C. albicans. (A) Galleria mellonella model of systemic infection. $2.5 \times 10^{5}$ cells of wild type (SC5314), complemented $(\mathrm{Cah} \times \mathrm{k} 2 \Delta / \Delta \mathrm{c} / \mathrm{C})$ or hexose kinase mutant strains were injected into the hemocoel at the last left-pro leg of $30 \mathrm{Galleria}$ larvae. Sterile $\mathrm{NaCl}(0.9 \%)$ was injected into control larvae. Survival was monitored for 8 days at $37^{\circ} \mathrm{C}$ and presented in a Kaplan-Meier plot. Statistical analysis was performed using log rank tests; ns, non-significant; ${ }^{* * *} P=0.0002 ;{ }^{* * * *} P<0.0001$. Cahxk2 $\Delta / \Delta$, Cahxk2glk1 $\Delta / \Delta$, and Caglk1glk4 $\Delta / \Delta$ mutant strains are significantly less virulent compared to the wild type strain $\left(P_{\text {value }} \leq 0.0001\right)$. The difference observed between Caglk $1 \Delta / \Delta$ mutant and the wild type strain is not significant $\left(P_{\text {value }}>0.05\right)$. The complemented strain exhibits higher virulence than hexokinase mutants but lesser than the wild type $\left(P_{\text {value }}=0.002\right)$. (B) Flow cytometer analysis of mouse macrophage interaction with live C. albicans cells in stationary phase at $\mathrm{MOI}$ 1:5 (1 macrophage for 5 yeasts) over a 24-h time course experiment. The horizontal bars represent the macrophage survival, indicated as a percentage on the left side of the bar. The white part of the bars represents the percentage of non-phagocytozing macrophages. The shades tones part represents the percentage of phagocytozing macrophages. Histograms represent a mean of three independent experiments performed on three different biological samples, repeated five times (+ standard deviation). (C) Representative pictures of the J774 macrophages after 4 and 24 h of infection with wild type and hexose kinase mutant strains in culture flasks at MOI 1:5. The scale bars represent $100 \mu \mathrm{m}$.

( $60 \%$ compared to approximately $80 \%$ for the other strains). This suggests that the absence of hexokinase or glucokinase has no impact on the recognition step. Survival of macrophages was severely higher when $\operatorname{Cah} x k 2 \Delta / \Delta$ and $\operatorname{Cah} x k 2 g l k 1 \Delta / \Delta$ were tested. After $4 \mathrm{~h}$ in the presence of the hexokinase mutant $(\operatorname{Cah} x k 2 \Delta / \Delta)$ the number of alive macrophages was nearly twice as high as in the presence of the wild type strain. Moreover, $90 \%$ of the macrophages infected by $\operatorname{Cah} x k 2 g l k 1 \Delta / \Delta$ were still alive after $4 \mathrm{~h}$, while $34 \%$ remained alive with the wild type strain. After $24 \mathrm{~h}, 39 \%$ of the macrophages infected with Cahxk2glk1 $\Delta / \Delta$ survived, compared to only $2 \%$ with the wild type strain and $1 \%$ for the other strains. This underlines again the very affected virulence capacities of this double mutant and highlights a role for Glk1 in the absence of Hxk2. Reintegration of the wild type CaHXK2 gene restored the killing capacities, suggesting that the virulence defect was linked to the absence of CaHXK2. As compared to the wild type strain, interactions performed with glucokinase mutants $(\operatorname{Caglk1} \Delta / \Delta$, Caglk $1 g l k 4 \Delta / \Delta)$ and macrophages did not reveal significant differences. Because the process of macrophage killing relies on the formation that pierce the phagocytic membrane, the morphogenesis of the strains was analyzed after 4 and $24 \mathrm{~h}$ of infection (Figure 7C). Our data clearly reveal that $C a h x k 2 \Delta / \Delta$ and $C a h x k 2 g l k 1 \Delta / \Delta$ did not develop hyphae during macrophage infection. In order to make 
sure that the growth defect of the hexokinase mutant strains was not the main cause for avirulence, $C$. albicans cells were released from macrophage after $4 \mathrm{~h}$ of phagocytosis by cell lysis and counted (Supplementary Figure S2). As compared to the wild type, there was no significant differences in the capacity of the mutant strains to divide inside the macrophage.

Altogether, these data suggest that the virulence defect associated to the deletion of Cahxk2 could concern the fungal escape phase rather than the recognition and initial phagocytosis step.

\section{DISCUSSION}

In this study, we sought to assign functions to the hexokinase and glucokinases that could potentially contribute to the fitness and virulence of $C$. albicans. We showed that hexose phosphorylation

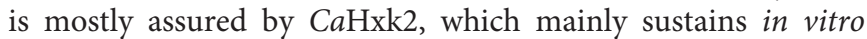
growth in the presence of hexoses. Hexokinase expression is induced by glucose and higher than glucokinase expression. But proteins are both detectable even in the absence of any phosphorylable hexose. As shown for S. cerevisiae glycolytic enzymes, regulation is the result of a complex mixture of gene expression and metabolic effects, in order to optimize simultaneously fluxes, protein and metabolite concentrations (Daran-Lapujade et al., 2007). C. albicans inhabits niches containing contrasting carbon sources. Metabolic flexibility implies that alternative carbon sources and glucose are assimilated simultaneously (Barelle et al., 2006; Childers et al., 2016). The discrepancy between C. albicans transcriptome and proteome has been already clearly highlighted (Sandai et al., 2012). Upon glucose exposure, CaIcl1 and CaPck1, enzymes involved in the assimilation of alternative carbon sources are not degraded, while their transcripts are subjected to glucose

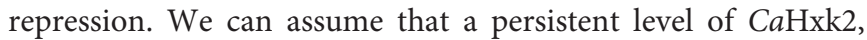
$\mathrm{CaGlk1}$ and $\mathrm{CaGlk} 4$ could promote metabolic flexibility and stress response to cope with changing microenvironments reached by the pathogen in the various host niches.

The affected growth profiles of $\operatorname{Cah} x k 2 \Delta / \Delta$ and Cahxk2glk1 $\Delta / \Delta$ indicate the limited ability of $C a \mathrm{Glk} 1$ and $\mathrm{CaGlk} 4$ to allow glucose utilization in the absence of $\mathrm{CaHxk} 2$, while normal growth was observed in the absence of CaGlk1 and/CaGlk4. One possible hypothesis would be a limited glucose uptake caused by the absence of hexokinase. Hence, in $S$. cerevisiae and $K$. lactis glycolytic mutants, glycolysis controls glucose signaling via the SRR pathway. The expression of several glucose-regulated genes, like hexose transporter genes, depends on a functional glycolysis, limiting therefore glucose import (Cairey-Remonnay et al., 2015). However, this control does not seem to exist in C. albicans. On the contrary, expression of transporter genes controlled by the SRR pathway (HGH12, HGT7, HXT10) was enhanced in the hexokinase mutant $\operatorname{Cah} x k 2 \Delta / \Delta$. Therefore, the poor expression of glucokinase genes, the low intracellular concentration of $\mathrm{CaGlk} 1$ and $\mathrm{CaGlk} 4$ and their low participation in hexose kinase activity, could mainly explain the growth defect in the absence of hexokinase.
Moreover, glycolysis constitutes an interface between metabolism and gene transcription. For instance, glycolysis yields pyruvate which can be oxidized into acetyl-CoA, directly implicated in histone acetylation and gene expression. In stationary yeast cells, increase glucose availability leads to higher levels of acetyl-CoA synthesis, global histone acetylation, accompanied by the induction of a thousand of growth-related genes (Friis et al., 2009). The reduced glycolytic flux of the hexokinase mutant could therefore lead to transcription defects and slower growth. In all the tested conditions, the double mutant Cahxk2glk1 $\Delta / \Delta$ presented an altered phenotype. In this context, $\mathrm{CaGlk} 4$ is the only hexose kinase enzyme present. Because of the low affinity of $\mathrm{CaGlk} 4$ for glucose and its very low expression, growth of Cahxk $2 g l k 1 \Delta / \Delta$ is drastically affected in the presence of hexoses. This could be explained by the lack of efficient hexose kinase enzymes. Moreover, hexokinase and glucokinase gene deletions could lead to several drastic intracellular changes. In $S$. cerevisiae, the $h x k 2$ mutant has a higher $\mathrm{H}^{+}$-ATPase activity and a lower pyruvate decarboxylase activity which coincided with an intracellular accumulation of pyruvate (Diderich et al., 2001). Absence of glucose repression could also contribute to redirect carbon flux. In $K$ lactis, the identification of hexokinase-dependent proteins related to chromatin remodeling, amino acids and protein metabolism, redox maintenance and stress response reinforces the idea that glucose kinase enzymes exert broader functions than hexose phosphorylation and glucose repression (Mates et al., 2014).

Our findings reveal that the well-established bifunctional functions of Hxk2 in S. cerevisiae (Herrero et al., 1995; Vega et al., 2016) also exists in C. albicans, while glucokinases do not seem to play a role in glucose repression. We detected $\mathrm{CaHxk2-GFP}$ in the nuclei in $0.1 \%$ glucose-grown cells $(5 \mathrm{mM})$, which corresponds to the glucose level maintained in the bloodstream and in vaginal secretions (Barelle et al., 2006; Brown et al., 2014). Glucose repression pathway via $\mathrm{CaHxk} 2$, could thereby promote metabolic adaptation to favor the fitness of the pathogen, even in glucose-limited host niches. In response to glucose and according to the $S$. cerevisiae model, $\mathrm{CaHxk} 2$ should act as a transcriptional carbon catabolite corepressor binding to CaMig1 (Ahuatzi et al., 2007). C. albicans has two orthologs of ScMig1, CaMig1 and CaMig2 but, to our knowledge, no functions have been assigned yet to CaMig2. Transcriptional studies realized on CaMig1 revealed that it regulates a unique set of genes, annotated as carbohydrate uptake and catabolism factors (Murad et al., 2001). However, works conducted on CaMig1 revealed that it has no phosphorylation sequence for the kinase CaSnf1, essential for the removal of glucose repression (Petter et al., 1997; Zaragoza et al., 2000). Deletion of CaMig1 has no effect on the expression of CaGAL1, a glucose repressed gene (Zaragoza et al., 2000) but impacts the transcription of hexose transporter genes (Broxton et al., 2018). Moreover, CaMig1 has been recently implicated in the resistance to weak organic acids, a novel function (Cottier et al., 2015). All these evidences show that some of the molecular mechanisms involved in glucose repression in C. albicans remain to be elucidated, in particular concerning the direct partners of $\mathrm{CaHxk2}$. Moreover, contrary to $S$. cerevisiae (Moreno and Herrero, 2002), C. albicans glucokinases are not 
subjected to glucose repression. This suggests that glucokinases are not involved into the control of glucose phosphorylation in C. albicans and underline their minor role in glucose metabolism.

Contrary to glucokinases, hexokinase gene deletion had an impact on various in vitro stress responses. Glucose has been shown to promote stress resistance and to induce some stress genes in C. albicans (Rodaki et al., 2009). Our data support this finding, but furthermore indicate a role for glucose phosphorylation in stress resistance. Osmotic and oxidative stresses induce storage of trehalose, glycerol, and arabitol (Sánchez-Fresneda et al., 2013). Biosynthesis of such osmolyte sugars and polyols, directly connected to the upper part of the glycolytic pathway, depends on glucose-6-phosphate availability. This was confirmed in S. cerevisiae where the analysis of metabolic fluxes in a $\Delta h x k 2$ mutant revealed a synthesis of glycerol reduced by a factor of 4.5 (Raghevendran et al., 2004). Moreover, the carbon source modulates cell wall architecture and strongly influences the resistance of $C$. albicans to osmotic and cell wall stresses. Glucose and lactate-grown cells display significant differences in cell wall elasticity and ultrastructure (Ene et al., 2012). $\beta$-Glucans are major constituents of $C$. albicans cell wall. Glucan synthases assemble UDP-glucose residues produced from phosphorylated glucose (Free, 2013). Thus, any hexose phosphorylation defect would affect the cell wall and render it more sensitive to stress.

Glycolytic gene expression has been associated to the global response of C. albicans to hypoxia (Synnott et al., 2010). Several transcription factors are involved in hypoxia-responsive expression of glycolytic genes. Among them, the key filamentation regulator Efg1 and the transcription factors Tye7 and Gal4 contribute to the early hypoxic response (Setiadi et al., 2006; Askew et al., 2009; Bonhomme et al., 2011; Sellam et al., 2014). Contrary to S. cerevisiae which ferments sugars even under aerobic conditions, C. albicans, a crabtree-negative yeast, ferments carbohydrates under hypoxic conditions (Johnston, 1999). Our findings specify a clear and drastic effect of hypoxia on glucokinase expression at the mRNA and protein levels. Glucokinase enzymes could be part of the global early hypoxic response, as a spare wheel, to maintain a necessary glycolytic flux during fermentation conditions in oxygen-poor niches. Moreover, this data confirms the fact that hexokinase and glucokinases are not targeted by the same regulatory pathways.

Our results show that the hexokinase mutant retains the filamentation capacity when the carbon source does not require $\mathrm{CaHxk} 2$ to be assimilated. Thus, the filamentation-defective phenotype of the hexokinase mutant could be linked to a phosphorylation defect. The absence of one or both glucokinases has no impact on filamentation. This could be related to their low contribution to hexose phosphorylation and, concerning the spider medium, because glucokinases do not phosphorylate fructose. The activities of several major glycolytic enzymes are known to differ in yeast and hyphal forms (Schwartz and Larsh, 1982). We have shown that induction of filamentation requires upregulation of hexose kinase genes. The morphological switch to filamentous growth requires energy and carbon source, notably to build membranes and cell walls. Thereby, several links between morphogenesis and expression of metabolic genes are established in C. albicans. The transcription factor Efg1, part of a Ras-cAMP-PKA signaling network and involved in morphogenesis in C. albicans, strongly induces glycolytic and fermentation genes (Doedt et al., 2004). Moreover, mutants of the $\mathrm{CaHgt} 4 / \mathrm{CaRgt} 1$ pathway (SRR pathway) involved in the control of gene expression in the absence of glucose display affected filamentation phenotypes (Brown et al., 2006; Sexton et al., 2007). Thus, nested pathways control simultaneously morphogenesis, glucose signaling and metabolism and by this way $\mathrm{CaHxk} 2$, which directly impacts on filamentation through its kinase activity.

The hypovirulence of the hexokinase mutant suggests a central function for $\mathrm{CaHxk2}$. We have shown that the glucose phosphorylation step controls filamentation. Morphological switch is a determining factor of virulence in both Galleria and macrophage models. Hyphae are observed in the G. mellonella infected tissues (Fuchs et al., 2010). Histological investigations of infected larvae revealed that the SC5314 wild type strain shows a high propensity to filament, leading to gut invasion (Perdoni et al., 2014). Time-lapse microscopy studies have shown a strong correlation between intra-phagocytic hyphal growth and macrophage lysis (Bain et al., 2015). Numerous experimental data support a model by which $C$. albicans hyphae enable escape from phagocytes by growing and consequently lysing the cell (Erwig and Gow, 2016). CaHxk2 could then be required to sustain filamentation within the host cell. Moreover, the impact could be situated at the metabolic level. C. albicans hexokinase and double glucokinase mutants could degrade trehalose, major hemolymph sugar in G. mellonella larvae (Shukla et al., 2015) to recover glucose, but remain unable or less efficient to phosphorylate it, respectively. Recently, Tucey et al. (2018) revealed concomitant up-regulation of host and pathogen glycolysis, setting up glucose competition by depleting glucose. C. albicans-activated macrophages shift to Warburg metabolism and become dependent on glucose for survival. During macrophage infection, both $C$. albicans free cells and escaped from macrophage could trigger rapid death to the phagocytes by depleting glucose levels. The hexokinase mutant could not compete efficiently for glucose and then turn out to be hypovirulent. Affected metabolic capacities of the hexokinase mutant, along with a less effective stress resistance and adaptation to hypoxic environments could impact colonization of host environments. When hexoses are naturally available like in the gastrointestinal tract, in the bloodstream or on epithelial cell surfaces, budding growth may depend on the optimal functioning of the hexokinase. Infection and invasion of cells, tissues and organs is associated to morphological plasticity of C. albicans. Infection hyphae actively adhere and penetrate into epithelial cells or induce their endocytic uptake (Dalle et al., 2010; Martin et al., 2011). Thus, filamentation defects of the mutant strain Cahxk2 could also impact on its invasion capacities. Barelle et al. (2006) showed, using GFP fusions, that glyoxylate cycle and gluconeogenic genes are repressed by physiologically relevant concentrations of glucose during kidney infection. Thus, C. albicans cells growing in the mouse kidney model are supposed 
to assimilate carbon via glycolytic metabolism. Consequently, a decreased glycolytic activity could also directly impact on the invasion and infection capacities of the pathogen.

Our data decipher the role of glucose kinase enzymes, not only as a central point of metabolism, but also as actors in regulation, stress response and morphogenesis. Altogether, those different interconnected functions influence the virulence of the yeast. Surprisingly, while the lack of glucokinases did not impact on the phenotype of the mutants, $\mathrm{CaGlk1}$ clearly appeared implicated in the hypoxic response. Moreover, the fact that hexose transporter gene expression level is affected in Caglk1glk $4 \Delta / \Delta$ suggests that glucokinases could be implicated in regulation processes that remain to be elucidated. Future research might provide further insights in this challenging area.

\section{AUTHOR CONTRIBUTIONS}

RL and PC designed and performed the experiments, analyzed the data, and wrote the manuscript. $\mathrm{KD}$ and $\mathrm{BD}$ performed the experiments, contributed to the manuscript writing and data

\section{REFERENCES}

Ahuatzi, D., Riera, A., Peláez, R., Herrero, P., and Moreno, F. (2007). Hxk2 regulates the phosphorylation state of Migl and therefore its nucleocytoplasmic distribution. J. Biol. Chem. 282, 4485-4493. doi: 10.1074/jbc.M606854200

Armstrong-James, D., Meintjes, G., and Brown, G. D. (2014). A neglected epidemic: fungal infections in HIV/AIDS. Trends Microbiol. 22, 120-127. doi: 10.1016/j.tim.2014.01.001

Askew, C., Sellam, A., Epp, E., Hogues, H., Mullick, A., Nantel, A., et al. (2009). Transcriptional regulation of carbohydrate metabolism in the human pathogen Candida albicans. PLoS Pathog. 5:e1000612. doi: 10.1371/journal.ppat.1000612

Bain, J., Gow, N. A. R., and Erwig, L.-P. (2015). Novel insights into host-fungal pathogen interactions derived from live-cell imaging. Semin. Immunopathol. 37 , 131-139. doi: 10.1007/s00281-014-0463-3

Barelle, C. J., Priest, C. L., Maccallum, D. M., Gow, N. A. R., Odds, F. C., and Brown, A. J. P. (2006). Niche-specific regulation of central metabolic pathways in a fungal pathogen. Cell. Microbiol. 8, 961-971. doi: 10.1111/j.1462-5822.2005. 00676.x

Bernardo, S. M. H., Gray, K.-A., Todd, R. B., Cheetham, B. F., and Katz, M. E. (2007). Characterization of regulatory non-catalytic hexokinases in Aspergillus nidulans. Mol. Genet. Genom. 277, 519-532. doi: 10.1007/s00438-006-0203-z

Biswas, S., Van Dijck, P., and Datta, A. (2007). Environmental sensing and signal transduction pathways regulating morphopathogenic determinants of Candida albicans. Microbiol. Mol. Biol. Rev. 71, 348-376. doi: 10.1128/MMBR.00009-06

Bonhomme, J., Chauvel, M., Goyard, S., Roux, P., Rossignol, T., and d'Enfert, C. (2011). Contribution of the glycolytic flux and hypoxia adaptation to efficient biofilm formation by Candida albicans. Mol. Microbiol. 80, 995-1013. doi: 10 . 1111/j.1365-2958.2011.07626.x

Bork, P., Sander, C., and Valencia, A. (1992). An ATPase domain common to prokaryotic cell cycle proteins, sugar kinases, actin, and hsp70 heat shock proteins. Proc. Natl. Acad. Sci. U.S.A. 89, 7290-7294. doi: 10.1073/pnas.89.16. 7290

Brown, A. J. P., Brown, G. D., Netea, M. G., and Gow, N. A. R. (2014). Metabolism impacts upon Candida immunogenicity and pathogenicity at multiple levels. Trends Microbiol. 22, 614-622. doi: 10.1016/j.tim.2014. 07.001

Brown, A. J. P., Odds, F. C., and Gow, N. A. R. (2007). Infection-related gene expression in Candida albicans. Curr. Opin. Microbiol. 10, 307-313. doi: 10. 1016/j.mib.2007.04.001

Brown, V., Sexton, J. A., and Johnston, M. (2006). A glucose sensor in Candida albicans. Eukaryot. Cell 5, 1726-1737. doi: 10.1128/EC.00186-06 analysis. AS, TN, and ML contributed to manuscript writing and data analysis.

\section{ACKNOWLEDGMENTS}

We are grateful to Jade Ravent for technical assistance. We thank J. Morschhäuser and S. Bates for providing the pSFS2A and pGFP-NAT1 plasmids, respectively. The RT-qPCR experiments were performed thanks to the DTAMB (Développement de Techniques et Analyse Moléculaire de la Biodiversité, Université Lyon 1). RL was the recipient of a fellowship from the Ministère de la Recherche of France. This work was released as a preprint at BioRxiv, https://doi.org/10.1101/448373, (Laurian et al., 2018).

\section{SUPPLEMENTARY MATERIAL}

The Supplementary Material for this article can be found online at: https://www.frontiersin.org/articles/10.3389/fmicb. 2019.00327/full\#supplementary-material

Broxton, C. N., He, B., Bruno, V. M., and Culotta, V. C. (2018). A role for Candida albicans superoxide dismutase enzymes in glucose signaling. Biochem. Biophys. Res. Commun. 495, 814-820. doi: 10.1016/j.bbrc.2017. 11.084

Cairey-Remonnay, A., Deffaud, J., Wésolowski-Louvel, M., Lemaire, M., and Soulard, A. (2015). Glycolysis controls plasma membrane glucose sensors to promote glucose signaling in yeasts. Mol. Cell. Biol. 35, 747-757. doi: 10.1128/ MCB.00515-14

Calderone, R. A., and Clancy, C. J. (2012). Candida and Candidiasis. Washington, DC: ASM Press.

Cárdenas, M. L., Cornish-Bowden, A., and Ureta, T. (1998). Evolution and regulatory role of the hexokinases. Biochim. Biophys. Acta 1401, 242-264. doi: 10.1016/S0167-4889(97)00150-X

Childers, D. S., Raziunaite, I., Mol Avelar, G., Mackie, J., Budge, S., Stead, D., et al. (2016). The rewiring of ubiquitination targets in a pathogenic yeast promotes metabolic flexibility, host colonization, and virulence. PLoS Pathog. 12:e1005566. doi: 10.1371/journal.ppat.1005566

Collart, M. A., and Oliviero, S. (2001). Preparation of yeast RNA. Curr. Protoc. Mol. Biol. 23, 13.12.1-13.12.5. doi: 10.1002/0471142727.mb1312s23

Corvey, C., Koetter, P., Beckhaus, T., Hack, J., Hofmann, S., Hampel, M., et al. (2005). Carbon Source-dependent assembly of the Snflp kinase complex in Candida albicans. J. Biol. Chem. 280, 25323-25330. doi: 10.1074/jbc. M503719200

Cottier, F., Tan, A. S. M., Xu, X., Wang, Y., and Pavelka, N. (2015). MIG1 regulates resistance of Candida albicans against the fungistatic effect of weak organic acids. Eukaryot. Cell 14, 1054-1061. doi: 10.1128/EC.00129-15

Dalle, F., Wächtler, B., L'Ollivier, C., Holland, G., Bannert, N., Wilson, D., et al. (2010). Cellular interactions of Candida albicans with human oral epithelial cells and enterocytes. Cell. Microbiol. 12, 248-271. doi: 10.1111/j.1462-5822. 2009.01394.x

Daran-Lapujade, P., Rossell, S., van Gulik, W. M., Luttik, M. A. H., de Groot, M. J. L., Slijper, M., et al. (2007). The fluxes through glycolytic enzymes in Saccharomyces cerevisiae are predominantly regulated at posttranscriptional levels. Proc. Natl. Acad. Sci. U.S.A. 104, 15753-15758. doi: 10.1073/pnas. 0707476104

Dementhon, K., El-Kirat-Chatel, S., and Noël, T. (2012). Development of an in vitro model for the multi-parametric quantification of the cellular interactions between Candida yeasts and phagocytes. PLoS One 7:e32621. doi: 10.1371/journal.pone.0032621

Diderich, J. A., Raamsdonk, L. M., Kruckeberg, A. L., Berden, J. A., and Van Dam, K. (2001). Physiological properties of Saccharomyces cerevisiae from 
which hexokinase II has been deleted. Appl. Environ. Microbiol. 67, 1587-1593. doi: 10.1128/AEM.67.4.1587-1593.2001

Doedt, T., Krishnamurthy, S., Bockmühl, D. P., Tebarth, B., Stempel, C., Russell, C. L., et al. (2004). APSES proteins regulate morphogenesis and metabolism in Candida albicans. Mol. Biol. Cell 15, 3167-3180. doi: 10.1091/mbc.e03-11-0782

Eisman, B., Alonso-Monge, R., Román, E., Arana, D., Nombela, C., and Pla, J. (2006). The Cek1 and Hogl mitogen-activated protein kinases play complementary roles in cell wall biogenesis and chlamydospore formation in the fungal pathogen Candida albicans. Eukaryot. Cell 5, 347-358. doi: 10.1128/ EC.5.2.347-358.2006

Ene, I. V., Adya, A. K., Wehmeier, S., Brand, A. C., MacCallum, D. M., Gow, N. A. R., et al. (2012). Host carbon sources modulate cell wall architecture, drug resistance and virulence in a fungal pathogen. Cell. Microbiol. 14, 1319-1335. doi: $10.1111 / j .1462-5822.2012 .01813 . x$

Ene, I. V., Cheng, S.-C., Netea, M. G., and Brown, A. J. P. (2013). Growth of Candida albicans cells on the physiologically relevant carbon source lactate affects their recognition and phagocytosis by immune cells. Infect. Immun. 81, 238-248. doi: 10.1128/IAI.01092-12

Erwig, L. P., and Gow, N. A. R. (2016). Interactions of fungal pathogens with phagocytes. Nat. Rev. Microbiol. 14, 163-176. doi: 10.1038/nrmicro.2015.21

Fan, J., Chaturvedi, V., and Shen, S.-H. (2002). Identification and phylogenetic analysis of a glucose transporter gene family from the human pathogenic yeast Candida albicans. J. Mol. Evol. 55, 336-346. doi: 10.1007/s00239-0022330-4

Fernández-García, P., Peláez, R., Herrero, P., and Moreno, F. (2012). Phosphorylation of yeast hexokinase 2 regulates its nucleocytoplasmic shuttling. J. Biol. Chem. 287, 42151-42164. doi: 10.1074/jbc.M112.401679

Flores, C. L., Rodríguez, C., Petit, T., and Gancedo, C. (2000). Carbohydrate and energy-yielding metabolism in non-conventional yeasts. FEMS Microbiol. Rev. 24, 507-529. doi: 10.1016/S0168-6445(00)00037-1

Free, S. J. (2013). Fungal cell wall organization and biosynthesis. Adv. Genet. 81, 33-82. doi: 10.1016/B978-0-12-407677-8.00002-6

Friis, R. M. N., Wu, B. P., Reinke, S. N., Hockman, D. J., Sykes, B. D., and Schultz, M. C. (2009). A glycolytic burst drives glucose induction of global histone acetylation by picNuA4 and SAGA. Nucleic Acids Res. 37, 3969-3980. doi: $10.1093 /$ nar/gkp270

Fuchs, B. B., Eby, J., Nobile, C. J., El Khoury, J. B., Mitchell, A. P., and Mylonakis, E. (2010). Role of filamentation in Galleria mellonella killing by Candida albicans. Microbes Infect. 12, 488-496. doi: 10.1016/j.micinf.2010.03.001

Gillum, A. M., Tsay, E. Y., and Kirsch, D. R. (1984). Isolation of the Candida albicans gene for orotidine-5'-phosphate decarboxylase by complementation of S. cerevisiae ura3 and E. coli pyrF mutations. Mol. Gen. Genet. 198, 179-182. doi: $10.1007 /$ BF00328721

Herrero, P., Galíndez, J., Ruiz, N., Martínez-Campa, C., and Moreno, F. (1995). Transcriptional regulation of the Saccharomyces cerevisiae HXK1, HXK2 and GLK1 genes. Yeast 11, 137-144. doi: 10.1002/yea.320110205

Jarvis, W. R., and Martone, W. J. (1992). Predominant pathogens in hospital infections. J. Antimicrob. Chemother. 29(Suppl. A), 19-24. doi: 10.1093/jac/29. suppl_A.19

Johnston, M. (1999). Feasting, fasting and fermenting. Glucose sensing in yeast and other cells. Trends Genet. 15, 29-33. doi: 10.1016/S0168-9525(98)01637-0

Kuser, P. R., Krauchenco, S., Antunes, O. A., and Polikarpov, I. (2000). The high resolution crystal structure of yeast hexokinase PII with the correct primary sequence provides new insights into its mechanism of action. J. Biol. Chem. 275, 20814-20821. doi: 10.1074/jbc.M910412199

Kuzmic, P. (1996). Program DYNAFIT for the analysis of enzyme kinetic data: application to HIV proteinase. Anal. Biochem. 237, 260-273. doi: 10.1006/abio. 1996.0238

Laurian, R., Dementhon, K., Doumèche, B., Soulard, A., Noel, T., Lemaire, M., et al. (2018). Hexokinase and glucokinases are essential for fitness and virulence in the pathogenic yeast Candida albicans. bioRXiv [Preprint]. doi: 10.1101/448373

Lorenz, M. C., Bender, J. A., and Fink, G. R. (2004). Transcriptional response of Candida albicans upon internalization by macrophages. Eukaryot. Cell 3, 1076-1087. doi: 10.1128/EC.3.5.1076-1087.2004

Lorenz, M. C., and Fink, G. R. (2001). The glyoxylate cycle is required for fungal virulence. Nature 412, 83-86. doi: 10.1038/35083594

Martin, R., Wächtler, B., Schaller, M., Wilson, D., and Hube, B. (2011). Hostpathogen interactions and virulence-associated genes during Candida albicans oral infections. Int. J. Med. Microbiol. 301, 417-422. doi: 10.1016/j.ijmm.2011. 04.009

Mates, N., Kettner, K., Heidenreich, F., Pursche, T., Migotti, R., Kahlert, G., et al. (2014). Proteomic and functional consequences of hexokinase deficiency in glucose-repressible Kluyveromyces lactis. Mol. Cell. Proteom. 13, 860-875. doi: $10.1074 /$ mcp.M113.032714

Milne, S. W., Cheetham, J., Lloyd, D., Aves, S., and Bates, S. (2011). Cassettes for PCR-mediated gene tagging in Candida albicans utilizing nourseothricin resistance. Yeast 28, 833-841. doi: 10.1002/yea.1910

Miramón, P., and Lorenz, M. C. (2017). A feast for Candida: metabolic plasticity confers an edge for virulence. PLoS Pathog. 13:e1006144. doi: 10.1371/journal. ppat.1006144

Moreno, F., Ahuatzi, D., Riera, A., Palomino, C. A., and Herrero, P. (2005). Glucose sensing through the Hxk2-dependent signalling pathway. Biochem. Soc. Trans. 33, 265-268. doi: 10.1042/BST0330265

Moreno, F., and Herrero, P. (2002). The hexokinase 2-dependent glucose signal transduction pathway of Saccharomyces cerevisiae. FEMS Microbiol. Rev. 26, 83-90. doi: 10.1111/j.1574-6976.2002.tb00600.x

Murad, A. M., d'Enfert, C., Gaillardin, C., Tournu, H., Tekaia, F., Talibi, D., et al. (2001). Transcript profiling in Candida albicans reveals new cellular functions for the transcriptional repressors CaTup1, CaMig1 and CaNrg1. Mol. Microbiol. 42, 981-993. doi: 10.1046/j.1365-2958.2001.02713.x

Noble, S. M., Gianetti, B. A., and Witchley, J. N. (2017). Candida albicans cell-type switching and functional plasticity in the mammalian host. Nat. Rev. Microbiol. 15, 96-108. doi: 10.1038/nrmicro.2016.157

Odds, F. C. (1988). Candida and Candidosis. London: Bailliere Tindall Press.

Perdoni, F., Falleni, M., Tosi, D., Cirasola, D., Romagnoli, S., Braidotti, P., et al. (2014). A histological procedure to study fungal infection in the wax moth Galleria mellonella. Eur. J. Histochem. 58:2428. doi: 10.4081/ejh.2014.2428

Pérez, J. C., Kumamoto, C. A., and Johnson, A. D. (2013). Candida albicans commensalism and pathogenicity are intertwined traits directed by a tightly knit transcriptional regulatory circuit. PLoS Biol. 11:e1001510. doi: 10.1371/ journal.pbio. 1001510

Perlroth, J., Choi, B., and Spellberg, B. (2007). Nosocomial fungal infections: epidemiology, diagnosis, and treatment. Med. Mycol. 45, 321-346. doi: 10.1080/ 13693780701218689

Petter, R., Chang, Y. C., and Kwon-Chung, K. J. (1997). A gene homologous to Saccharomyces cerevisiae SNF1 appears to be essential for the viability of Candida albicans. Infect. Immun. 65, 4909-4917.

Raghevendran, V., Gombert, A. K., Christensen, B., Kötter, P., and Nielsen, J. (2004). Phenotypic characterization of glucose repression mutants of Saccharomyces cerevisiae using experiments with 13C-labelled glucose. Yeast 21, 769-779. doi: 10.1002/yea.1136

Randez-Gil, F., Herrero, P., Sanz, P., Prieto, J. A., and Moreno, F. (1998). Hexokinase PII has a double cytosolic-nuclear localisation in Saccharomyces cerevisiae. FEBS Lett. 425, 475-478. doi: 10.1016/S0014-5793(98)00289-0

Rao, K. H., Ghosh, S., Natarajan, K., and Datta, A. (2013). N-acetylglucosamine kinase, HXK1 is involved in morphogenetic transition and metabolic gene expression in Candida albicans. PLoS One 8:e53638. doi: 10.1371/journal.pone. 0053638

Reuss, O., Vik, A., Kolter, R., and Morschhäuser, J. (2004). The SAT1 flipper, an optimized tool for gene disruption in Candida albicans. Gene 341, 119-127. doi: 10.1016/j.gene.2004.06.021

Rodaki, A., Bohovych, I. M., Enjalbert, B., Young, T., Odds, F. C., Gow, N. A. R., et al. (2009). Glucose promotes stress resistance in the fungal pathogen Candida albicans. Mol. Biol. Cell 20, 4845-4855. doi: 10.1091/mbc.e09-01-0002

Rolland, F., Wanke, V., Cauwenberg, L., Ma, P., Boles, E., Vanoni, M., et al. (2001). The role of hexose transport and phosphorylation in cAMP signalling in the yeast Saccharomyces cerevisiae. FEMS Yeast Res. 1, 33-45.

Rolland, S., Hnatova, M., Lemaire, M., Leal-Sanchez, J., and WésolowskiLouvel, M. (2006). Connection between the Rag4 glucose sensor and the KlRgt1 repressor in Kluyveromyces lactis. Genetics 174, 617-626. doi: 10.1534/genetics. 106.059766

Sabina, J., and Brown, V. (2009). Glucose sensing network in Candida albicans: a sweet spot for fungal morphogenesis. Eukaryot. Cell 8, 1314-1320. doi: 10.1128/ EC.00138-09

Sánchez-Fresneda, R., Guirao-Abad, J. P., Argüelles, A., González-Párraga, P., Valentín, E., and Argüelles, J.-C. (2013). Specific stress-induced storage of 
trehalose, glycerol and D-arabitol in response to oxidative and osmotic stress in Candida albicans. Biochem. Biophys. Res. Commun. 430, 1334-1339. doi: 10.1016/j.bbrc.2012.10.118

Sandai, D., Yin, Z., Selway, L., Stead, D., Walker, J., Leach, M. D., et al. (2012). The evolutionary rewiring of ubiquitination targets has reprogrammed the regulation of carbon assimilation in the pathogenic yeast Candida albicans. MBio 6:e2489-14. doi: 10.1128/mBio.00495-12

Schwartz, D. S., and Larsh, H. W. (1982). Comparative activities of glycolytic enzymes in yeast and mycelial forms of Candida albicans. Mycopathologia 78, 93-98. doi: 10.1007/BF00442632

Sellam, A., van het Hoog, M., Tebbji, F., Beaurepaire, C., Whiteway, M., and Nantel, A. (2014). Modeling the transcriptional regulatory network that controls the early hypoxic response in Candida albicans. Eukaryot. Cell 13, 675-690. doi: 10.1128/EC.00292-13

Setiadi, E. R., Doedt, T., Cottier, F., Noffz, C., and Ernst, J. F. (2006). Transcriptional response of Candida albicans to hypoxia: linkage of oxygen sensing and Efglp-regulatory networks. J. Mol. Biol. 361, 399-411. doi: 10.1016/j.jmb.2006. 06.040

Sexton, J. A., Brown, V., and Johnston, M. (2007). Regulation of sugar transport and metabolism by the Candida albicans Rgt1 transcriptional repressor. Yeast 24, 847-860. doi: 10.1002/yea.1514

Shukla, E., Thorat, L. J., Nath, B. B., and Gaikwad, S. M. (2015). Insect trehalase: physiological significance and potential applications. Glycobiology 25, 357-367. doi: 10.1093/glycob/cwu125

Synnott, J. M., Guida, A., Mulhern-Haughey, S., Higgins, D. G., and Butler, G. (2010). Regulation of the hypoxic response in Candida albicans. Eukaryot. Cell 9, 1734-1746. doi: 10.1128/EC.00159-10

Tucey, T. M., Verma, J., Harrison, P. F., Snelgrove, S. L., Lo, T. L., Scherer, A. K., et al. (2018). Glucose homeostasis is important for immune cell viability during Candida challenge and host survival of systemic fungal infection. Cell Metab. 27, 988-1006.e7. doi: 10.1016/j.cmet.2018.03.019
Vega, M., Riera, A., Fernández-Cid, A., Herrero, P., and Moreno, F. (2016). Hexokinase 2 is an intracellular glucose sensor of yeast cells that maintains the structure and activity of Mig1 protein repressor complex. J. Biol. Chem. 291, 7267-7285. doi: 10.1074/jbc.M115.711408

Walther, A., and Wendland, J. (2003). An improved transformation protocol for the human fungal pathogen Candida albicans. Curr. Genet. 42, 339-343. doi: 10.1007/s00294-002-0349-0

Wilson, D., Thewes, S., Zakikhany, K., Fradin, C., Albrecht, A., Almeida, R., et al. (2009). Identifying infection-associated genes of Candida albicans in the postgenomic era. FEMS Yeast Res. 9, 688-700. doi: 10.1111/j.1567-1364.2009. 00524.x

Yamada-Okabe, T., Sakamori, Y., Mio, T., and Yamada-Okabe, H. (2001). Identification and characterization of the genes for $\mathrm{N}$-acetylglucosamine kinase and $\mathrm{N}$-acetylglucosamine-phosphate deacetylase in the pathogenic fungus Candida albicans. Eur. J. Biochem. 268, 2498-2505. doi: 10.1046/j.1432-1327. 2001.02135.x

Zaragoza, O., Rodríguez, C., and Gancedo, C. (2000). Isolation of the MIG1 gene from Candida albicans and effects of its disruption on catabolite repression. J. Bacteriol. 182, 320-326. doi: 10.1128/JB.182.2.320-326.2000

Conflict of Interest Statement: The authors declare that the research was conducted in the absence of any commercial or financial relationships that could be construed as a potential conflict of interest.

Copyright (c) 2019 Laurian, Dementhon, Doumèche, Soulard, Noel, Lemaire and Cotton. This is an open-access article distributed under the terms of the Creative Commons Attribution License (CC BY). The use, distribution or reproduction in other forums is permitted, provided the original author(s) and the copyright owner(s) are credited and that the original publication in this journal is cited, in accordance with accepted academic practice. No use, distribution or reproduction is permitted which does not comply with these terms. 